\title{
A stochastic flow rule for granular materials
}

\author{
Ken Kamrin and Martin Z. Bazant \\ Department of Mathematics, Massachusetts Institute of Technology, Cambridge, MA 01239
}

(Dated: October 31, 2018)

\begin{abstract}
There have been many attempts to derive continuum models for dense granular flow, but a general theory is still lacking. Here, we start with Mohr-Coulomb plasticity for quasi-2D granular materials to calculate (average) stresses and slip planes, but we propose a "stochastic flow rule" (SFR) to replace the principle of coaxiality in classical plasticity. The SFR takes into account two crucial features of granular materials - discreteness and randomness - via diffusing "spots" of local fluidization, which act as carriers of plasticity. We postulate that spots perform random walks biased along slip-lines with a drift direction determined by the stress imbalance upon a local switch from static to dynamic friction. In the continuum limit (based on a Fokker-Planck equation for the spot concentration), this simple model is able to predict a variety of granular flow profiles in flat-bottom silos, annular Couette cells, flowing heaps, and plate-dragging experiments - with essentially no fitting parameters - although it is only expected to function where material is at incipient failure and slip-lines are inadmissible. For special cases of admissible slip-lines, such as plate dragging under a heavy load or flow down an inclined plane, we postulate a transition to rate-dependent Bagnold rheology, where flow occurs by sliding shear planes. With different yield criteria, the SFR provides a general framework for multiscale modeling of plasticity in amorphous materials, cycling between continuum limit-state stress calculations, meso-scale spot random walks, and microscopic particle relaxation.
\end{abstract}

\section{INTRODUCTION}

For centuries, engineers have described granular materials using continuum solid mechanics [1, 2, 3]. Dense granular materials behave like rigid solids at rest, and yet are easily set into liquid-like, quasi-steady motion by gravity or moving boundaries, so the classical theory is Mohr-Coulomb plasticity (MCP), which assumes a frictional yield criterion. The simplest model is the two-dimensional "Ideal Coulomb Material" at limit-state, where the maximum ratio of shear to normal stress is everywhere equal to a constant (the internal friction coefficient), whether or not flow is occurring. This model is believed to describe stresses well in static or flowing granular materials, but, as we explain below, it fails to predict flow profiles, when combined with the usual Coaxial Flow Rule of continuum plasticity. Indeed, it seems continuum mechanics has not yet produced a simple and robust model for granular flow.

In recent years, the sense that there is new physics to be discovered has attracted a growing community of physicists to the study of granular materials [4, 5, $, \mathbf{6}, \mathbf{7}$, 8, 9]. Unlike the engineers, their interest is mostly at the discrete particle level, motivated by the breakdown of classical statistical mechanics and hydrodynamics due to strong dissipation and long-lasting, frictional contact networks. Dense granular materials exhibit many interesting collective phenomena, such as force chains, slow structural relaxation, and jamming. Similar non-equilibrium phenomena occur in glasses, foams, and emulsions, as in granular materials, so it is hoped that a general new statistical theory may emerge. Presumably from such a microscopic basis, continuum models of glassy relaxation and dense granular flow could be systematically derived, just as dissipative hydrodynamics for granular gases can be derived from kinetic theory with inelastic collisions [10].

This dream has not yet been achieved, but many empirical continuum models have been proposed [4, 9, 11]. The difficulty in describing dense granular flow is evidenced by the remarkable diversity of physical postulates, which include: coupled static and rolling phases 12, 13, 14, 15]. Bagnold rheology 16] based on "granular eddies" [17], granular temperature-dependent viscosity [18], density-dependent viscosity [19, 20], non-local stress propagation along arches 21], self-activated shear events due to non-local stress fluctuations 22, 23], freevolume diffusion opposing gravity 24, 25, 26, 27, 28], "shear transformation zones" coupled to free-volume kinetics [29, 30], and partial fluidization governed by a Landau-like order parameter [31, 32]. Each of these theories can fit a subset of the experimental data [33], usually only for a specific geometry for which it was designed, such as a flowing surface layer $12,13,14,15,31]$, inclined plane 16, 17], Couette cell 19, 20], inclined chute [22, 23], or wide silo [24, 25, 26, 27, 28], and none seems to have very broad applicability. For example, we are not aware of a single model, from physics or engineering, which can predict velocity profiles in both draining silos and annular Couette cells, even qualitatively.

The theory of partial fluidization of Aranson and Tsimring has arguably had the most success in describing multiple flows within a single theoretical framework [31, 32]. Although setting boundary conditions for the order parameter usually requires additional $a d$ hoc assertions, the model is nonetheless able to reproduce known flow behavior in inclined chutes, avalanches, rotating drums, and simple shear cells without many fitting parameters. It also describes some unsteady flows. However, the theory lacks any clear microscopic foundation and is not directly 
coupled to a constitutive stress model for static materials. As such, it has only been applied to problems with very simple solid stress fields, limiting its current applicability to flows that depend on only one spatial variable.

In an attempt to describe arbitrary geometries, such as silos and Couette cells, we take the view that the engineers may already have a reasonable continuum description of the mean stresses, so we start with Mohr-Coulomb plasticity. However, discreteness and randomness clearly need to be taken into account in a granular material. For static stresses, quenched randomness in material properties is known to lead to statistical slip-line blurring in "stochastic plasticity" 34], but this says nothing about how plastic yielding actually occurs.

To describe yielding dynamics, we propose a "stochastic flow rule" (SFR) where local fluidization (stick-slip transition) propagates randomly along blurred slip-lines. We build on the recently proposed Spot Model for random-packing dynamics 27] by viewing "spots" of free volume as carriers of plasticity in granular materials, analogous to dislocations in crystals. Multiscale spot simulations can reproduce quite realistic flowing packings in silo drainage [28]; here, we introduce a mechanical basis for spot motion from MCP, which leads to a theory of considerable generality for bulk granular flows.

The paper is organized as follows. Since plasticity is unfamiliar to most physicists, we begin by reviewing key concepts from MCP in section III both for stresses and for dense flows. In section [II] we highlight various shortcomings of the classical theory, many of which we attribute to the Coaxial Flow Rule. We then introduce the general spot-based SFR and a specific simplification to be used for granular flow in section IV Next we apply the theory to four prototypical examples: silo, Couette, heap, and plate-dragging flows in section $\nabla$ Then in section VI we explain how the last two examples indicate a smooth transition from the SFR to Bagnold rheology, when slip-lines become admissible, and we present a simple composite theory, which extends the applicability of the model to various shear flows. In section VII] we conclude by further clarifying the range of applicability of the SFR and possible extensions to other granular flows and different materials.

\section{CONCEPTS FROM CONTINUUM MECHANICS}

\section{A. Mohr-Coulomb plasticity: stresses}

In the eighteenth century, it was Coulomb, as a military engineer designing earthen fortresses, who introduced the classical model of a granular material, which persists to the present day: a continuous medium with a frictional yield criterion. His ideas were expressed in general continuum-mechanical terms by Mohr a century later, and a modern mathematical formulation of "MohrCoulomb plasticity" (MCP), which we also use below, is

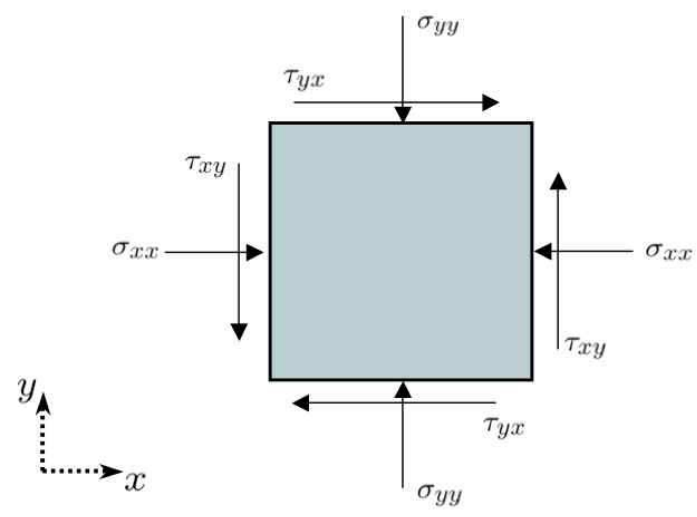

FIG. 1: Stresses on a material element. All vectors are pointing in the positive direction as per our sign convention.

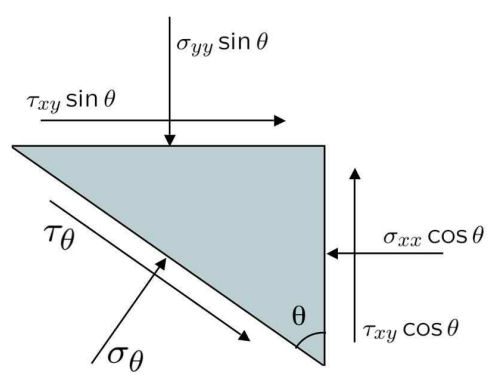

FIG. 2: Force diagram for a wedge. Hypotenuse length assigned to unity.

due to Sokolovskii [2]. Although other mechanical models exist, such as Drucker-Prager plasticity [35], MCP is perhaps the simplest and most widely used for granular materials in engineering [1]. As such, we choose to build our model of dense granular flow on the MCP description of stresses, as a reasonable and time-tested first approximation.

We begin in this section by reviewing relevant concepts from MCP, e.g. following Nedderman [1]. The fundamental assumption is that a granular material can be treated as an "Ideal Coulomb Material" (ICM), i.e. a rigid-plastic continuous media which yields according to a Coulomb yield criterion

$$
|\tau / \sigma|=\mu \equiv \tan \phi
$$

where $\tau$ is the shear stress, $\sigma$ is normal stress, and $\phi$ the internal friction angle, akin to a standard friction law with no cohesion. Throughout, we accept the common tensorial conventions for stresses with the key exception that normal stresses are deemed positive in compression. This is a standard modification in the study of non-cohesive granular materials since granular assemblies cannot support tension. We will also focus entirely on quasi-2D geometries.

Consider a small material element in static equilibrium and with no body forces present (see Figure1). The nor- 
mal stresses $\sigma_{x x}$ and $\sigma_{y y}$ can differ and the shear stresses $\tau_{x y}$ and $\tau_{y x}$ must be equal in order to balance moments. Likewise the variable $\tau_{y x}$ is redundant and will not be used again in this paper. To determine the stresses along any angle within this element, we place a new boundary within the material at some desired angle $\theta$ and observe force balance on the wedge that remains (see Figure 2). After algebraic simplification, this gives

$$
\begin{aligned}
\sigma_{\theta} & =\frac{1}{2}\left(\sigma_{x x}+\sigma_{y y}\right)+\frac{1}{2}\left(\sigma_{x x}-\sigma_{y y}\right) \cos 2 \theta-\tau_{x y} \sin 2 \theta \\
\tau_{\theta} & =\frac{1}{2}\left(\sigma_{x x}-\sigma_{y y}\right) \sin 2 \theta+\tau_{x y} \cos 2 \theta
\end{aligned}
$$

Now define

$$
\begin{aligned}
p & =\frac{1}{2}\left(\sigma_{x x}+\sigma_{y y}\right) \\
\tan 2 \psi & =\frac{-2 \tau_{x y}}{\sigma_{x x}-\sigma_{y y}} \\
R & =\sqrt{\left(\frac{\sigma_{x x}-\sigma_{y y}}{2}\right)^{2}+\tau_{x y}^{2}}
\end{aligned}
$$

which allows us to write

$$
\begin{aligned}
\sigma_{\theta} & =p+R \cos (2 \theta-2 \psi) \\
\tau_{\theta} & =R \sin (2 \theta-2 \psi)
\end{aligned}
$$

This implies that for all angles $\theta$, the locus of traction stresses $\left(\sigma_{\theta}, \tau_{\theta}\right)$ is a circle centered at $(p, 0)$ with radius $R$. This circle is referred to as "Mohr's Circle".

We have just derived Mohr's Circle without accounting for the possible effects of body forces acting on the material element and gradients in the stress field. Adjusting for these effects, however, would change the results only negligibly as the element gets small in size. If we were to apply the same force-balancing analysis to a differentially small material element with a body force and stress gradients, we would find that the stress differences on the walls and the inclusion of the differentially small body force within only add differentially small terms to the equations for $\sigma_{\theta}$ and $\tau_{\theta}$. Thus we can always use Mohr's Circle to obtain traction stresses along a desired angle.

To ultimately define a full stress state for the material element, we need one more equation - we have 3 stress variables and only 2 force balance equations:

$$
\begin{aligned}
& \frac{\partial \sigma_{x x}}{\partial x}-\frac{\partial \tau_{x y}}{\partial y}=F_{b o d y}^{x} \\
& \frac{\partial \sigma_{y y}}{\partial y}-\frac{\partial \tau_{x y}}{\partial x}=F_{b o d y}^{y}
\end{aligned}
$$

We say a material element is at incipient failure if the yield criterion is fulfilled along some direction and $|\tau / \sigma| \leq \mu$ along all others. A material in which incipient failure occurs everwhere is said to be at a limit-state. In a limit-state, the Mohr's Circle at every point in the material must be tangent to the locus $|\tau / \sigma|=\mu$. As can be seen by applying trigonometry in Figure 3 this requirement means that $R=p \sin \phi$, enabling us to parameterize the stresses in terms of $p$ and $\psi$ only, thereby closing the equations. For this reason, we restrain our anlysis to limit-state materials and refer to $p$ and $\psi$ as the stress parameters or Sokolovskii variables. (The limit-state stress treatment described here is also known as "Slip-Line Theory"; to avoid possible confusion, we specify this is not equivalent to Limit Analysis Plasticity concerned with upper and lower collapse limits.)

Solving for the original stress variables in terms of the stress parameters gives:

$$
\begin{aligned}
\sigma_{x x} & =p(1+\sin \phi \cos 2 \psi) \\
\sigma_{y y} & =p(1-\sin \phi \cos 2 \psi) \\
\tau_{x y} & =-p \sin \phi \sin 2 \psi
\end{aligned}
$$

Using these expressions, we re-write equations (4) and (5):

$$
\begin{aligned}
& (1+\sin \phi \cos 2 \psi) p_{x}-2 p \sin \phi \sin 2 \psi \psi_{x}+\sin \phi \sin 2 \psi p_{y} \\
& \quad+2 p \sin \phi \cos 2 \psi \psi_{y}=F_{\text {body }}^{x} \\
& \sin \phi \sin 2 \psi p_{x}+2 p \sin \phi \cos 2 \psi \psi_{x}+(1-\sin \phi \cos 2 \psi) p_{y} \\
& \quad+2 p \sin \phi \sin 2 \psi \psi_{y}=F_{b o d y}^{y}
\end{aligned}
$$

These will be referred to as the "stress balance equations". They form a hyperbolic system and thus can be solved using the method of characteristics. The system reduces to the following two characteristic equations:

$$
\begin{gathered}
d p \mp 2 p \mu d \psi=F_{b o d y}^{y}(d y \mp \mu d x)+F_{b o d y}^{x}(d x \pm \mu d y) \\
\text { along curves fulfilling } \frac{d y}{d x}=\tan (\psi \mp \epsilon) .
\end{gathered}
$$

To solve the stress balance equations, mesh the two families of characteristic curves in the bulk, then march from the boundaries in, progressively applying the two differential relationships above to approximate the stress parameters at each intersection point in the mesh. More on this can be found in [36. Other ways to solve the stress balance equations include the Two-Step Lax-Wendroff Method [37] and the Galerkin Method 38].

We return now to Mohr's Circle for a discussion of the stress properties within a differential material element. Equations (2) and (3) show that Mohr's Circle can be used as a slide-rule to determine the stresses along any angle $\theta$ : One arrives at the point $\left(\sigma_{\theta}, \tau_{\theta}\right)$ by starting at $\left(\sigma_{x x}, \tau_{x y}\right)$ and traveling anti-clockwise around Mohr's Circle for $2 \theta$ radians (see Figure 3). Also note on the diagram that the stresses along the $x$ and $y$ directions lie along a diameter of Mohr's Circle; any two material directions differing by an angle of $\pi / 2$ lie along a diameter of the corresponding Mohr's Circle diagram. Utilizing this property in reverse is perhaps the easiest way to draw Mohr's Circle in the first place; draw the unique circle for which $\left(\sigma_{x x}, \tau_{x y}\right)$ and $\left(\sigma_{y y},-\tau_{x y}\right)$ are endpoints of a diameter.

Let $\left(\sigma_{1}, 0\right)$ and $\left(\sigma_{3}, 0\right)$ be the points of intersection between Mohr's Circle and the $\sigma$-axis, where $\sigma_{1}>\sigma_{3}$. 


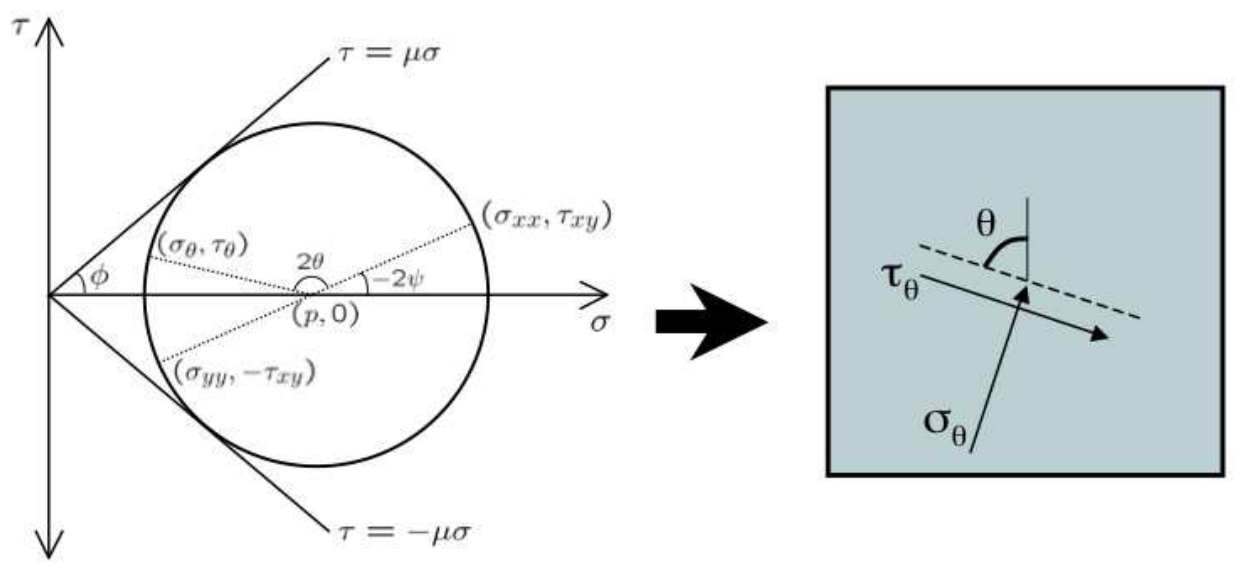

FIG. 3: Using Mohr's circle jointly with the Coulomb internal yield locus $(\tau= \pm \mu \sigma)$ to determine the traction stresses along any plane within a material element.

These points correspond to the two lines within a material element along which the shear stress vanishes and the normal stress is maximal or minimal. $\sigma_{1}\left(\sigma_{3}\right)$ is called the major (minor) principal stress and the line on which it acts is called the major (minor) principal plane.

Mohr's Circle shows that the major principal plane occurs at an angle $\psi$ anti-clockwise from the vertical (see Figure 31). Thus the major principal stress points along an angle $\psi$ anti-clockwise from the horizontal. This is the standard physical interpretation of $\psi$. One might think of $\psi$ as the angle from the horizontal along which a force chain would be predicted to lie.

By right-triangle geometry, a line segment connecting the center of Mohr's Circle to a point of tangency with the internal yield locus would make an angle of $\pi / 2-\phi$ with the $\sigma$-axis. Each point of tangency represents a direction along which the yield criterion is met, i.e. a slip-line. Mohr's Circle indicates that the slip-lines are angled $(\pi / 2-\phi) / 2$ up and down from the minor principal plane. But since the major and minor principal planes are orthogonal, the major principal stress points along the minor principal plane. Defining $\epsilon=\pi / 4-\phi / 2$, we deduce that slip-lines occur along the angles $\psi \pm \epsilon$ measured anti-clockwise from the from the horizontal. Looking back at the characteristic equations, we see that the slip-lines and the characteristic curves coincide. This means that information from the boundary conditions propagates along the slip-lines to form a full solution to the stress balance equations.

It is worth noting that the stress balance equations are written for static materials and do not appear to account for dynamic behavior like dilatancy and convection stresses. The theory of critical state soil mechanics [39] was the first to rigorously approach the issue of dilatancy (see appendix). It concludes that when material attains a flow state in which the density field stops changing in time, all points in the flow lie along a critical state line of the form $|\tau / \sigma|=\delta$ for $\delta$ constant. Since this exactly mirrors the Coulomb yield criterion, we can keep the stress

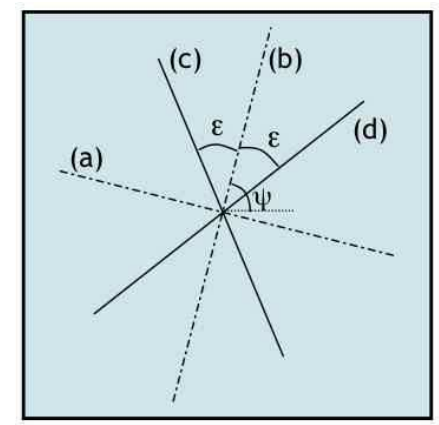

FIG. 4: Important lines intersecting each material point: (a) Major principal plane / Minor principal stress direction; (b) Minor principal plane / Major principal stress direction; (c)(d) Slip-lines.

balance equations and utilize $\delta=\mu$ (as in [40]). As for convection, adding the $\rho \mathbf{u} \cdot \nabla \mathbf{u}$ term into the stress equations couples the stresses to the velocity and makes the problem very difficult to solve. The practice of ignoring convection is justified by our slow-flow requirement and is commonly used and validated in basic solid mechanics literature [1, 2, 3]. So we conclude that dynamic effects in flowing materials do not preclude the use of the stress balance equations in slow, steady flows.

\section{B. Mohr-Coulomb plasticity: flow rules}

To calculate flow, we assert incompressibility and a flow rule - the flow rule is a constitutive law chosen to reflect the general behavior of the material at hand. The continuous nature of the ICM assumption suggests that symmetry should be kept with respect to the principal stress planes. Based on this, Jenike proposed adopting the Coaxial Flow Rule. The principle of coaxiality claims that material should flow by extending along the minor principal stress direction and contracting along the 


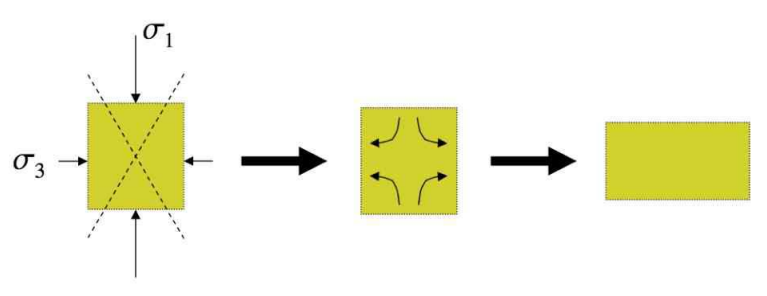

FIG. 5: Sketch of the Coaxial Flow Rule.

major principal stress direction; the principal planes of stress are aligned with the principal planes of strain-rate. The intuition for this constitutive rule is shown in Figure [5] Mathematically, this means that in a reference frame where the minor and major principal stress directions are the basis, the strain-rate tensor should have no off-diagonal components, i.e.

$$
\mathbf{R}_{\psi} \dot{\mathbf{E}} \mathbf{R}_{\psi}^{T} \text { is diagonal, }
$$

where $\mathbf{R}_{\psi}$ rotates anti-clockwise by $\psi$ and $\dot{\mathbf{E}}$ is the strainrate tensor

$$
\dot{\mathbf{E}}=\frac{1}{2}\left(\nabla \mathbf{u}+(\nabla \mathbf{u})^{T}\right) .
$$

where $\mathbf{u}=(u, v)$ is the velocity. Calculating the $(1,2)$ component of the matrix in equation (10) and setting it to zero gives the equation of coaxiality,

$$
\frac{\partial u}{\partial y}+\frac{\partial v}{\partial x}=\left(\frac{\partial u}{\partial x}-\frac{\partial v}{\partial y}\right) \tan 2 \psi
$$

This flow rule has played a dominant role in the development of continuum plasticity theory and will be closely analyzed in this work.

Coaxiality with incompressibility comprises another hyperbolic system of equations enabling the velocity field to be solved via characteristics:

$$
\begin{aligned}
& d u+\tan (\psi \mp \pi / 4) d v=0 \\
& \quad \text { along curves fulfilling } \frac{d y}{d x}=\tan (\psi \mp \pi / 4) .
\end{aligned}
$$

So, given $\psi(x, y)$ from the stress balance equations, information about the flow travels from the boundaries into the bulk along curves rotated $\pi / 4$ off from the principal stress planes - using Mohr's Circle, we observe that these are the lines for which the shear stress is maximal (and the normal stresses are equal).

Other flow rules have been suggested instead of coaxilaity. Of specific note, A.J.M. Spencer 41] has proposed the double-shearing flow rule. Unlike coaxiality which can be understood as a simultaneous equal shearing along both slip-line families, double-shearing allows the shearing motion to be unequally distributed between the two families in such a way that the flow remains isochoric.
For steady flows, the double-shearing flow rule is

$$
\begin{aligned}
& \sin 2 \psi\left(\frac{\partial u}{\partial x}-\frac{\partial v}{\partial y}\right)-\cos 2 \psi\left(\frac{\partial v}{\partial x}+\frac{\partial u}{\partial y}\right) \\
= & \sin \phi\left(\frac{\partial v}{\partial x}-\frac{\partial u}{\partial y}-2 \mathbf{u} \cdot \nabla \psi\right)
\end{aligned}
$$

It can be seen that when the material neighboring a particle rotates in sync with the rotation of the principal planes (i.e. as tracked by the rate of change of $\psi$ ), the right side goes to zero and the rule matches coaxiality. Under double-shearing, the characteristics of stress and velocity align, easing many aspects of the numerics. Some recent implementations of granular plasticity have utilized principles of double-shearing [42]. Though in this paper we deal primarily with the comparison of coaxiality to our new theory, this equation will be mentioned again in a later section.

\section{The rate-independence concept}

We now more fully address the conceptual basis for the flow theory just introduced. The theory is fundamentally different from traditional fluids where force-balance (including convection and viscous stresses in the case of Newtonian fluids) can be used alongside incompressibility to fully determine the fluid velocity and pressure fields. Unlike a fluid, granular materials can support a static shear stress and thus force-balance plus incompressibility alone is an underconstrained system. Rather, the stressstrain relationship for granular material is presumed to be rate-independent in the slow, quasi-static regime we study.

This concept is best understood tensorially. We can rewrite the equations of coaxiality and incompressibility equivalently as:

$$
\dot{\mathbf{E}}=\lambda \mathbf{T}_{\mathbf{0}}
$$

where

$$
\begin{array}{r}
\mathbf{T}=\text { Stress tensor }=\left(\begin{array}{cc}
-\sigma_{x x} & \tau_{x y} \\
\tau_{x y} & -\sigma_{y y}
\end{array}\right) \\
\mathbf{T}_{\mathbf{0}}=\mathbf{T}-\frac{1}{2}(\operatorname{tr} \mathbf{T}) \mathbf{I}=\text { Deviatoric stress tensor, }
\end{array}
$$

and $\lambda$ is a multiplier which can vary in space. Equation (12) is merely the ratio of the $(1,2)$ component and the difference of the $(2,2)$ and $(1,1)$ components of equation (15), thus cancelling $\lambda$, and incompressibility is automatic since we relate to the deviatoric stress tensor. Equation (15) gives a simple and highly general form for plastic material deformation applicable to a broad range of deformable materials and so it is ideal for illustrating the role of rate-dependency. In MCP, we solve for $\mathbf{T}_{\mathbf{0}}$ a priori from the stress balance equations. $\lambda$ adds the extra degree of freedom necessary to make sure the strain-rate field is actually compatible with a real velocity field- 
$\lambda$ is not any specific function of the stress or strain-rate variables and it adjusts to fit different velocity boundary conditions. Thus the stress alone does not imply the strain-rate and vice versa.

Supposing on the other hand that we were dealing with a rate-dependent (i.e. visco-plastic) material like Newtonian fluid, the above tensorial equation would still apply but we cannot claim to know $\mathbf{T}_{\mathbf{0}}$ in advance since material motion changes the stresses. Instead we prescribe a functional form for $\lambda$, like $\lambda=$ viscosity $^{-1}=$ constant, and write the force balance equations in terms of $\dot{\mathbf{E}}$. Thus $\dot{\mathbf{E}}$ is computed very differently for the two cases: in the rate-independent case, (15) is solved using a known form for $\mathbf{T}_{\mathbf{0}}$ and in the rate-dependent case, (15) is solved using a known form for $\lambda$.

The physical intuition for rate-independent flow can be easily understood with an example. Suppose we slide two frictional blocks against each other at two different non-zero sliding rates. In most rudimentry dry friction laws, the shear stress required to slide one block against another is proportional to the normal stress - there is no mention whatsoever of the rate of sliding. Thus the two sliding rates are modeled to be attainable with the same shear stress and likewise the stress-strain relationship is deemed rate-independent. For slow granular flows with long-lasting interparticle contacts, comparisons with this example are especially instructive.

\section{SHORTCOMINGS OF MOHR-COULOMB PLASTICITY}

The use of the stress balance equations with incompressibility and the Coaxial Flow Rule will be referred hitherto as Mohr-Coulomb Plasticity (MCP). The theory has the benefit of being founded on mechanical principles, but does have some marked drawbacks. We point out a few:

- The theory frequently predicts highly discontinuous velocity fields.

- The Coaxial Flow Rule is conceptually troubling in some simple geometries.

- The assumption of limit-state stresses is overreaching.

- MCP is a continuum theory and thus cannot model discreteness and randomness.

We will now discuss these four points in detail.

\section{A. Discontinuous "shocks" in stress and velocity}

The two stress PDEs and two flow PDEs are each fully hyperbolic systems meaning that continuous solutions do

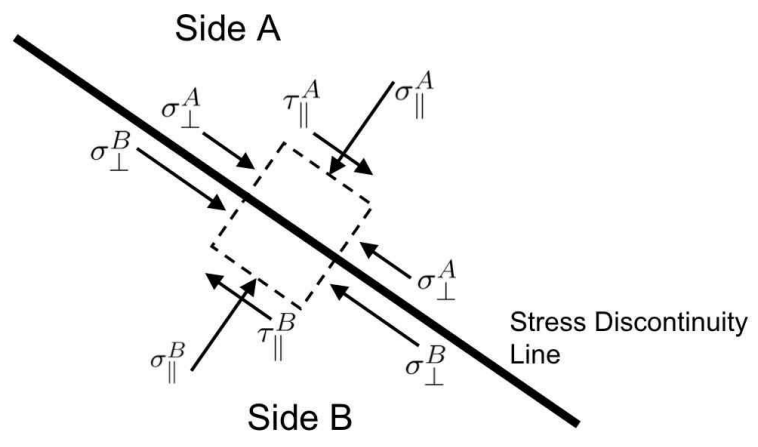

FIG. 6: Stresses on a control volume intersected by a discontinuity. Note how a jump in $\sigma_{\perp}$ places no net force on the control volume.

not necessarily exist for arbitrary choices of the boundary conditions. Instead, discontinuous solutions are constructed utilizing intuitive jump conditions. For stresses, a jump in the stress parameters across a discontinuity line is only allowable if such a jump places no net forces on a small control volume surrounding the line thereby ensuring particle stability. This means the normal and shear stresses along the direction of the discontinuity must be the same on both sides of the discontinuity. However, the normal stress along the perpendicular direction can have a jump upon crossing the discontinuity as this places no net force on the control volume (see Figure 6).

In terms of the stress parameters, this means that $p$ and $\psi$ can jump as long as

$$
\frac{\left(1+\sin \phi \cos \left(2 \Theta-2 \psi^{B}\right)\right)}{\left(1+\sin \phi \cos \left(2 \Theta-2 \psi^{A}\right)\right)}=\frac{\sin \left(2 \Theta-2 \psi^{B}\right)}{\sin \left(2 \Theta-2 \psi^{A}\right)}=\frac{p^{A}}{p^{B}}
$$

where $\Theta$ is the angle from the vertical along which the stress discontinuity lies.

As for velocity, incompressibility forces us to impose a simpler jump rule in that the component tangent to the velocity discontinuity is the only one allowed to jump. We note that whenever a stress shock exists, the jump in the stress parameters will usually place a jump in the flow rule and may cause a velocity discontinuity to form coincident with the stress shock. A velocity discontinuity can form even when the stress field is smooth since the velocity PDEs are themselves hyperbolic. (It is worth noting that when shocks are allowed in the solution, multiple solutions sometimes arise to the same problem; introduction of the so-called "entropy condition" can be used to choose the best of the possible solutions [1, 43].) Overall, the MCP equations are mathematically very poorly behaved, and have also been shown to give violent instabilities and finite-time singularities [44, 45].

Aside from its mathematical difficulties, MCP theory also does not match experiments or our everyday experience of granular flows. In particular, MCP commonly predicts complicated patterns of velocity discontinuities in situations where experiments indicate smooth flow in steady-state. In Figure 7 the numerically determined 


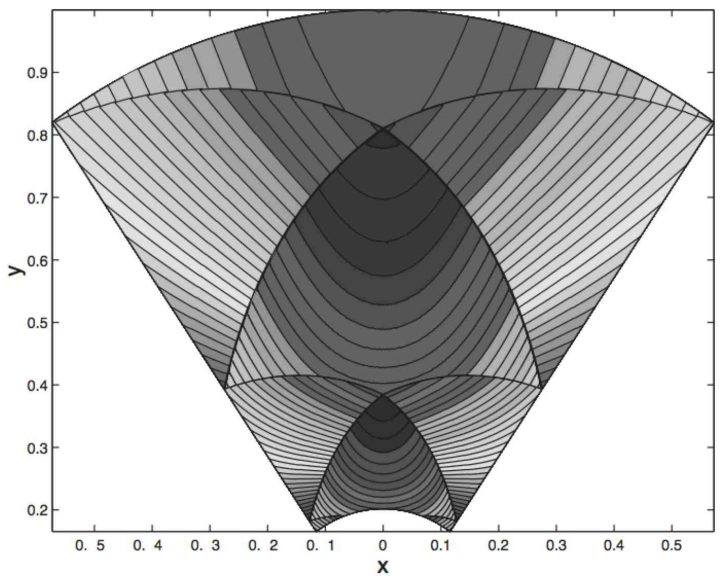

FIG. 7: Numerical solution to MCP in a wedge hopper with non-radial stresses on the top boundary. Normal stress in the radial direction displayed. (Courtesy of the authors of Ref. [38].)

stress field for a wedge hopper with only slightly nonradial boundary conditions on the top surface exhibits a fan-like array of shocks. The associated velocity field (not shown) will at best exhibit a similar pattern of discontinuities and at worse add even more discontinuities. Such a broken velocity field is clearly unphysical. As the grain size becomes very small (sands), discontinuous velocity fields with no relationship to the stress field have been observed, but these are only temporary; the shocks commonly blur away immediately after the onset of flow, which has been attributed to some instability mechanism [46]. Literature on the topic [1] is quick to concede that infinitessimally sharp velocity jumps are physically nonsensical and should be understood as being spread over at least a few particle widths. Below, we will see that our model naturally provides a mechanism for the blurring of velocity shocks even in the presence of a stress shock, with large velocity variations occurring only at the scale of several particle diameters.

Typically, to avoid the task of having to track/capture shocks in the stress/velocity field, approximations to MCP are invoked which give continuous solutions either by altering the boundary conditions or simplifying the PDE's. Smooth stress approximations are especially useful when attempting to solve for the velocity fieldtracking flow shocks coming from both a discontinuous stress field and hyperbolicity in the velocity equations is an enormous job. To our knowledge, a full solution to MCP has never been obtained either numerically or analytically in cases where the underlying stress field has shocks. Instead, shock-free approximate solutions have mostly been pursued.

Arguably, the two most successful and commonly used results of MCP are actually approximations, not full solutions. The Jenike Radial Solution 40, 47] for wedge hopper flow solves the MCP equations exactly, and with no discontinuities, but does not allow for a traction free top surface. It is a similarity solution of the form

$$
\begin{array}{r}
p=r f(\theta) \\
\psi=g(\theta) \\
\vec{v}=-\frac{h(\theta)}{r} \hat{r}
\end{array}
$$

which reduces the entire system to 3 ODE's with $(r, \theta)$ the position ( $r=$ distance from the hopper apex and $\theta$ is measured anticlockwise from the vertical). Though this solution enables the material to obey a wall yield criterion along the hopper walls, the stress parameters at the top surface have very little freedom. This is why most claim the Radial Solution to only hold near the orifice, considerably away from the actual top surface.

Another commonly used simplification is called the Method of Differential Slices, although it only applies to stresses and not flow (our focus here). Originally proposed by Janssen in 1895 and significantly enhanced since then, it is used to determine wall stresses in bins and containers. The method makes some very far-reaching assumptions about the internal stresses: $p$ is presumed to only depend on height and the $\psi$ field is assumed to be identically $\pi / 2$ or 0 . These assumptions reduce the stress balance equations to one ODE and ultimately give the famous result that wall stresses increase up to a certain depth and then saturate to a constant value. (This saturation behavior is not a byproduct of the approximation; the discontinuous, full solution to the stress balance equations in a bin also gives similar stress saturation behavior.) While this effect has been verified extensively in experiment, the underlying assumptions clearly cannot hold since, for example, the walls exert an upward shear stress on the material which contradicts the assumption about $\psi$. [1].

In summary, the equations of MCP theory have very limited applicability to granular flows. There are very few, if any, solutions available (either numerical or analytical) for many important geometries such as planar or annular Couette cells, vertical chutes, inclined places, etc. In the case of silos, MCP has been used extensively to describe stresses, although the equations are difficult to solve and poorly behaved from a mathematical point of view, as noted above. There have also been some attempts to use MCP to describe granular drainage from silos, in conjunction with the coaxial flow rule, but this approach has met with little success, as we now elaborate.

\section{B. Physical difficulties with coaxiality}

It is instructive to review the existing picture of silo drainage in MCP theory, to highlight what we will view as a major concern in the use of coaxiality for granular flows. Suppose we have a flat-bottomed quasi-2D silo with smooth side-walls. Under standard filling procedures, the walls provide only enough pressure to keep 
(a)

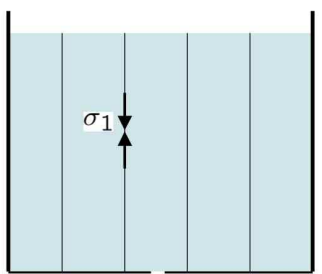

FIG. 8: Major principal stress chains in a quasi-2D silo for the (a) active case, and (b) passive case.

particles from sliding farther out. These wall conditions, known as the "active case", give the following solution to the stress balance equations as found by marching down characteristics starting from the flat, pressure free, top surface:

$$
\begin{aligned}
& \psi(x, y)=\pi / 2 \\
& p(x, y)=\frac{f_{g} y}{1+\sin \phi}
\end{aligned}
$$

where $f_{g} \equiv \rho g$ is the weight density of the material and $y$ is positive downward. Since the $\psi$ field is identically $\pi / 2$ everywhere, the slip-lines are thus perfectly straight lines angled at $\pm \epsilon$ from the vertical.

Refer again to Figure 5 The material deforms based solely on principal plane alignment. For a slow, dense flow in the silo geometry, coaxiality is troubling. Since the major principal stress is everywhere vertical, coaxiality requires material to stretch horizontally making it geometrically impossible for the material to converge and exit through the silo orifice. This issue has been dodged historically by asserting that a drastic change in the wall stresses occurs once the orifice opens, such that the walls drive the flow, not gravity [1]. The silo supposedly enters a "passive state" where the walls are pushing so hard on the material that the material is literally squeezed through the orifice by the walls. Even with this heavyhanded presumption, the solution predicted by equation (12) is absurd; it predicts the only non-stagnant regions in the silo are two narrow, straight channels which converge on the silo opening and are angled at $\pm 45^{\circ}$ from the vertical.

Coaxiality also suffers thermodynamically. The equation itself only ensures there is no shear strain-rate in the principal stress reference frame and actually does not directly enforce that of the two principal strain-rate axes, the axis of maximal compression (i.e. the major principal strain-rate direction) must align with the major principal stress direction, as was the physical intuition shown in Figure 5 . Coaxiality just as easily admits solutions for which the minor principal stresses align with the major principal strain-rate. When this happens, the plastic power dissipated per unit volume can be written

$$
\begin{aligned}
P & =\mathbf{T}: \dot{\mathbf{E}} \\
& =\left(\begin{array}{cc}
-\sigma_{1} & 0 \\
0 & -\sigma_{3}
\end{array}\right):\left(\begin{array}{cc}
|\dot{\gamma}| & 0 \\
0 & -|\dot{\gamma}|
\end{array}\right) \\
& =|\dot{\gamma}|\left(\sigma_{3}-\sigma_{1}\right) \\
& <0
\end{aligned}
$$

where $\mathbf{A}: \mathbf{B}=\Sigma A_{i j} B_{i j}$ and $\pm|\dot{\gamma}|$ are the principal strain-rate values. This type of behavior violates the second law of thermodynamics as it implies that material deformation does work on the system and likewise is non-dissipative. In more advanced plasticity theories, the thermodynamic inequality is upheld by requiring $\lambda$ in equation (15) to be non-negative, but in the basic limitstate framework we discuss, this contraint cannot be directly enforced.

We should quickly mention that in constructing the limit-state stress field for the discharging silo, we have used as a boundary condition that flow ensues when the pressure $p$ above the hole drops differentially from the value it takes when the hole is closed. This claim allows us to preserve the continuous stress field described in equations (22) and (23) for slow, quasi-static flow.

\section{Incipient yield everywhere}

The constraint of assuming a limit-state stress field of incipient yield everywhere is also questionable. Granular flows can contain regions below the yield criterion within which the plastic strain-rate must drop to zero. For example in drainage from a wide silo with a small orifice, the lower regions far from the orifice are completely stagnant [48, 49], and thus can hardly be considered to be at incipient yield. In fact, discrete-element simulations show that grains in this region essentially do not move from their initial, static packing [28]. Simulations also reveal that high above the orifice, where the shear rate is reduced, the packing again becomes nearly rigid [50], suggesting that the yield criterion is not met there either. As the silo example illustrates, a more general description of stresses coming from an elasto-plasticity theory may be necessary to properly account for sub-yield material [3, 51].

Elasto-plasticity also alleviates another major concern with MCP which is that it is only well-defined in two dimensions. Three-dimensional stress tensors have six free variables, too many to be uniquely described by just force balance and incipient failure (altogether only 4 equations). We mention in passing that extensions of $\mathrm{MCP}$ to axisymmetric three-dimensional situations have been developed. For example, the Har Von Karman hypothesis, which assumes that the intermediate principal stress $\sigma_{2}$, where $\sigma_{1} \leq \sigma_{2} \leq \sigma_{3}$, is identical to either $\sigma_{1}$ or $\sigma_{3}$, is frequently utilized in solving for conical hopper flow. However, elasto-plasticity can handle three dimensions without this hypothesis, while also allowing for 


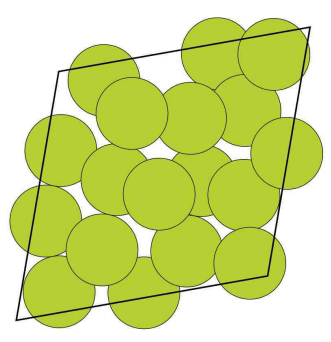

FIG. 9: A meso-scale object containing a small number of randomly packed, discrete grains, which controls the dynamics of dense granular flow, analogous to the "representative volume element" in classical continuum mechanics.

stress states below the yield criterion in different regions.

\section{Neglect of discreteness and randomness}

Beyond its practical limitations and mathematical difficulties, the most basic shortcomings of MCP are in its assumptions. Above all, a granular material is not continuous. The microscopic grains composing it are usually visible to the naked eye, and significant variations in the velocity often occur across a distance of only several particle lengths, e.g. in shear bands and boundary layers. Of course, the general theory of deterministic continuum mechanics is only expected to apply accurately when the system can be broken into "Representative Volume Elements" (RVE's) of size $L$ fulfilling $d \ll L \ll L_{\text {macro }}$ for $d$ the microscale (particle size) and $L_{\text {macro }}$ the size of the system [52], which is clearly violated in many granular flows. Therefore, the discrete, random nature of the particle packing must play an important role in the deformation process. To incorporate this notion coherently, it may be useful to seek out a dominant meso-scale object as a substitute for the RVE, upon which mechanical flow ideas apply, but in a non-deterministic, stochastic fashion (see Figure 9). This concept is somewhat comparable to the "Stochastic Volume Element" in the theory of plasticity of heterogeneous materials 34]. In that setting, it is known that (what physicists would call "quenched") randomness in material properties leads to blurring of the slip-lines, but, to our knowledge, this effect has not been considered in MCP for granular materials.

More importantly, however, since the meso-scale should only be a few grains in width, there must also be randomness in the dynamics of yielding to an applied stress or body force, since the theoretical concept of a continuous slip-line is incompatible with the reality of a discrete, random packing. A stochastic response also seems fundamentally more consistent with the assumption of inicipient yield: If the material is just barely in equilibrium, it must be very sensitive to small, random fluctuations, causing localized yielding.

We conclude that the shortcomings of MCP may be related to the deterministic Coaxial Flow Rule, so we now proceed to replace it with a more physically appropriate Stochastic Flow Rule. The Mohr-Coulomb description of stresses is more clearly grounded in principles of solid mechanics and is widely used in silo engineering, so we will assume that it still holds, on average, in the presence of slow flows, as a first approximation.

\section{THE STOCHASTIC FLOW RULE}

\section{A. Diffusing "spots" of plastic deformation}

It has been noted in a variety of experiments that dense granular flows can have velocity profiles which ressemble solutions to a diffusion equation. By far the best example is drainage from a wide silo, which has a wellknown Gaussian profile near the orifice, spreading vertically as the square root of the height (with parabolic streamlines) in a range of experiments [26, 48, 49, 53, 54]. Recently, experiments in the split-bottom Couette geometry have demonstrated precise error-function profiles of the velocity spreading upward from the shearing circle, albeit with more complicated scaling [55]. Shear bands, when they exist, tend to be exponentially localized near moving rough walls, but we note that these too can be viewed as solutions to a steady drift-diffusion equation with drift directed toward the wall.

It seems, therefore, that a successful flow rule for dense granular materials could be based on a stochastic process of deformation, consistent with our general arguments above based on discreteness and randomness. This begs the question: What is the diffusing carrier of plastic deformation? In crystals, plasticity is carried by dislocations, but it is not clear that any such defects might exist in an amorphous material. The Gaussian velocity profile of granular drainage was first explained independently by Litwiniszyn [24, 56] and Mullins [25] as being due to the diffusion of voids upward from the orifice, exchanging with particles to cause downward motion. However, this model cannot be taken literally, since granular flows have nearly uniform density with essentially no voids and with far less cage-breaking than the model would predict.

Instead, the starting point for our theory lies in the work of Bazant 27], who proposed a general model for the flow of amorphous materials (dense random packings) based on diffusing "spots" of cooperative relaxation, as illustrated in Figure 10. The basic idea is that each random spot displacement causes a small block-like displacement of particles in the opposite direction. This flow mechanism takes into account the tendency of each particle to move together with its cage of first neighbors, so the size of a spot is typically three to five particle diameters, $L_{s} \approx 3-5 d$. This expectation has been confirmed in dense silo drainage as the length scale for spatial velocity correlations in the experiments of Choi et al. [49, 57] (data shown in Figure 11) as well as the discrete-element simulations of Rycroft et al. 28]. In the continuum me- 
(a)

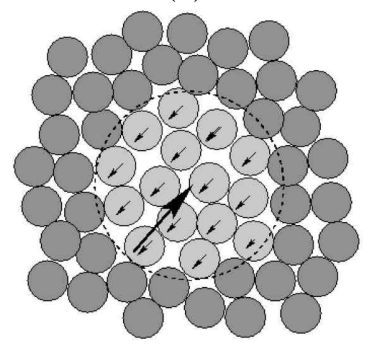

(b)

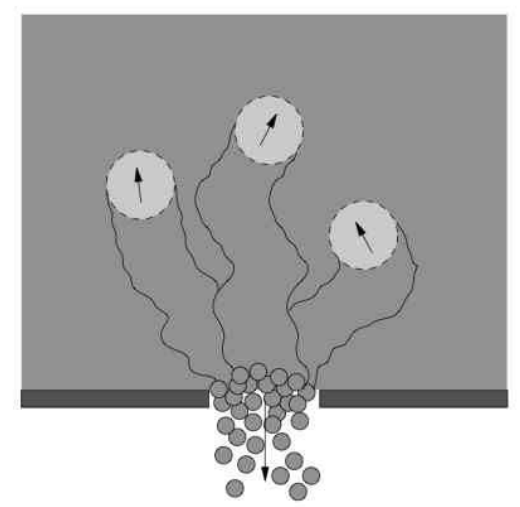

(c)

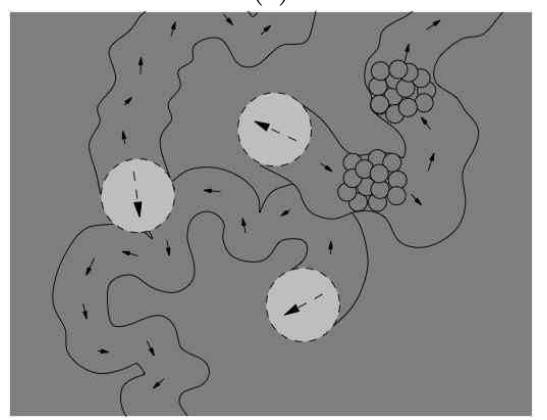

FIG. 10: Spots as carriers of plastic deformation in amorphous materials. (a) Cartoon of basic spot motion. A spot of local fluidization, carrying some free volume, moves to the upper right causes a cooperative displacement of particles, on average to the lower left, opposing the spot displacement. (b) In silo drainage, spots are injected at the orifice and perform random walks biased upward by gravity, causing downward motion of particles. (c) In other situations, we conjecture that spots are created during initial shear dilation and perform random walks biased by local stress imbalances and body forces during steady flow.

chanics terminology, this suggest that the spot may be an appropriate meso-scale replacement for the RVE.

A major motivation for our work comes from the recent demonstration by Rycroft et al. that the Spot Model can be used as a basis for realistic multiscale simulations of dense granular drainage in a wide silo, assuming that spots perform upward random walks, biased uniformly by gravity [28]. Using only five fitting parameters (the size, volume, diffusivity, drift speed, and creation rate of
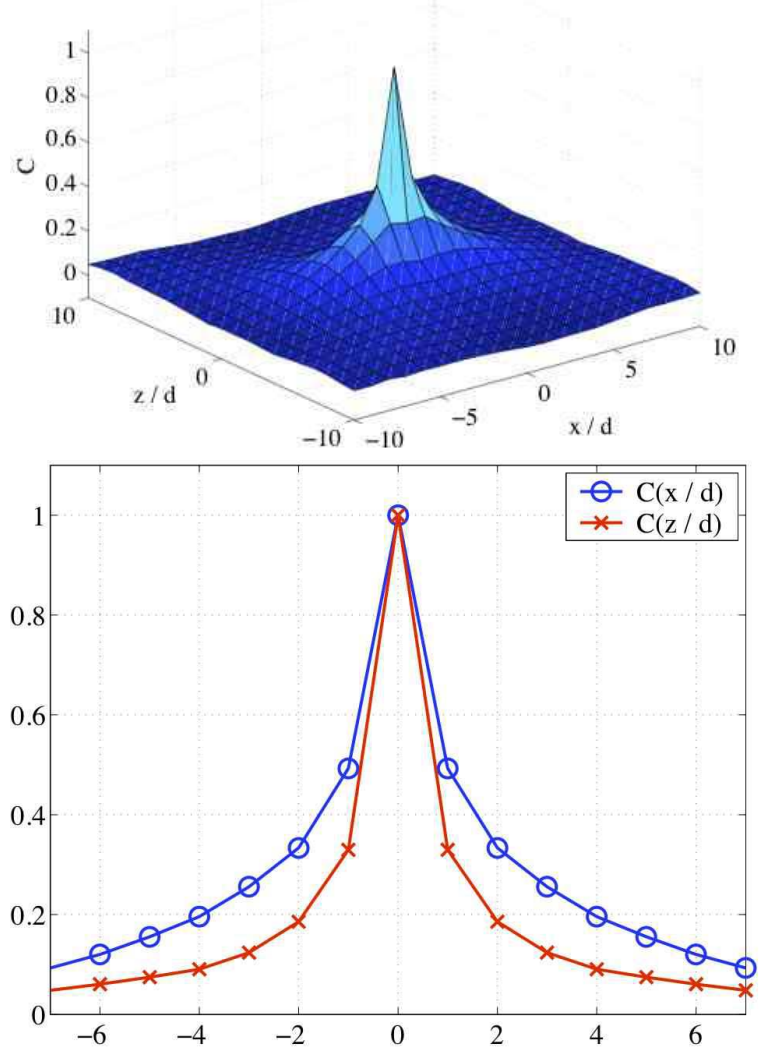

FIG. 11: Spatial velocity correlations in silo drainage experiments as in Refs. [49, 57] with glass beads $(d=3 \mathrm{~mm})$ obtained high-speed digital-video particle tracking. The correlation coefficient of instantaneous displacements of a pair different particles is plotted as a function of their separation, averaged over all pairs and all times in the video. (Courtesy of the authors of Ref. [57].)

spots), the spot simulations were able to accurately reproduce the statistical dynamics of several hundred thousand frictional, visco-elastic spheres in discrete-element simulations of drainage from a wide silo. This suggests that a general understanding of dense granular flows may come from a mechanical theory of spot dynamics.

\section{B. General form of the flow rule}

In this work, we propose such a mechanical theory, based on the assumption that MCP provides a reasonable description of the mean stresses in slow dense granular flows. The key idea is to replace the Coaxial Flow Rule with a "Stochastic Flow Rule" based on mechanically biased spot diffusion. In the continuum approximation, the general form of the flow rule thus consists of two steps 27]: (i) a Fokker-Planck (drift-diffusion) equation is solved for the probability density (or concentration) of 
spots, $\rho_{s}(\mathbf{r}, t)$ :

$$
\frac{\partial \rho_{s}}{\partial t}+\frac{\partial}{\partial x^{\alpha}}\left(D_{1}^{\alpha} \rho_{s}\right)=\frac{\partial}{\partial x^{\alpha}} \frac{\partial}{\partial x^{\beta}}\left(D_{2}^{\alpha \beta} \rho_{s}\right)
$$

where $\left\{D_{1}^{\alpha}\right\}$ is the drift vector and $\left\{D_{2}^{\alpha \beta}\right\}$ the diffusivity tensor of spots, determined by the stress field (below); and (ii) the mean drift velocity of particles $\mathbf{u}=\left\{u^{\alpha}\right\}$ is constructed to oppose the local flux of spots:

$$
\begin{aligned}
u^{\alpha}= & -\int d \mathbf{r}^{\prime} w\left(\mathbf{r}, \mathbf{r}^{\prime}\right)\left[D_{1}^{\alpha}\left(\mathbf{r}^{\prime}, t\right) \rho_{s}\left(\mathbf{r}^{\prime}, t\right)\right. \\
& \left.-\frac{\partial}{\partial x^{\beta}}\left(D_{2}^{\alpha \beta}\left(\mathbf{r}^{\prime}, t\right) \rho_{s}\left(\mathbf{r}^{\prime}, t\right)\right)\right]
\end{aligned}
$$

where $w\left(\mathbf{r}, \mathbf{r}^{\prime}\right)$ is the (dimensionless) spot "influence function" specifying how much a particle at $\mathbf{r}$ moves in response to a spot displacement at $\mathbf{r}^{\prime}$. Without making the continuum approximation, the same physical picture can also be the basis for a multiscale model, alternating between macroscopic continuum stress computation and discrete spot-driven random-packing dynamics 28].

The mean flow profile (25) is derived from a nonlocal stochastic partial differential equation for spot-driven particle dynamics, in the approximation that spots do not interact with each other [27]. Here, we have assumed the centered Stratonovich definition of stochastic differentials [58], which means physically that the spot influence is centered on its displacement. In contrast, Bazant used the one-sided reverse-Itto definition, where the spot influence is centered on the end of its displacement, which leads to an extra factor of two in the last term [27]. This choice is mathematically unrestricted (the "stochastic dilemna" 58]), but we find the centered definition to be a somewhat more reasonable physical hypothesis. Rycroft et al. have also found that the centered definition produces more realistic flowing random packings in multiscale spot simulations of granular drainage, when compared to full discrete-element simulations [28]. If we use the simple approximation $w \approx \delta\left(\left|\mathbf{r}-\mathbf{r}^{\prime}\right|\right)$ in the integral for particle velocity, the Stratonovich interpretation has the benefit of automatically upholding volume conservation.

Without even specifying how local stresses determine spot dynamics, the general form of the flow rule (25) predicts continuous velocity fields, even when the mean stresses are discontinuous. For example, shocks in the MCP stress field may lead to discontinuities in the spot drift vector, $\mathbf{D}_{1}$, and thus the spot flux. However, due to the nonlocal nature of the spot model, the particle flux is a convolution of the spot flux with the spot influence function, thus preserving a continuous velocity field, which varies at scales larger than the spot size, $L_{s}$. This is a direct consequence of the geometry of dense random packings: The strong tendency for particles to move with their nearest neighbors smears velocity changes over at least one correlation length.

In the simplest approximation, the spot influence is translationally invariant, $w=w\left(\mathbf{r}-\mathbf{r}^{\prime}\right)$, so that spots everywhere in the system have the same size and shape. The spot influence decays off for $r>L_{s}$, as a Gaussian among other possibilities.

In (25) we allow for the likelihood that the spot influence may not be translationally and rotationally invariant, e.g. since the local stress state always breaks symmetry. This is actually clear in the experimental measurements of Figure 11, where velocity correlations are more short-ranged, without roughly half the decay length, in the vertical direction (parallel to gravity) compared to the horizontal (transverse) direction. This suggests that spots are generally non-spherical and may be more elongated in the directions transverse to their drift (or the body force). If anisotropy in the spot influence were taken into account, it would also be natural to allow for an anisotropic spot diffusivity tensor, which depends on the local stress state. However, such effects seem to be small in the granular flows we consider below, which are well described by a much simpler model.

Another likely possibility is that spots come in a range of shapes and sizes. There could be a statistical distribution of regions of local fluidization or plastic yield related to the local packing and stress state. It is thus more reasonable to think of the spot influence function $w\left(\mathbf{r}, \mathbf{r}^{\prime}\right)$ as averaging over this distribution, just as a spatial velocity correlation measurement averages over a large number of collective relaxations. One advantage of taking the continuum limit of a Fokker-Planck equation (24) in applying the SFR is that such details are buried in the coefficients, which could in in principle be derived systematically from any microscopic statistical model, or simply viewed as a starting point for further physical hypotheses (as we do below).

Finally, we mention that there are also surely some nontrivial interactions between spots, which would make the SFR nonlinear and could lead to interesting new phenomena, such as spontaneous pattern formation. For example, spots may have a medium range attraction, since it is more difficult to propagate particle rearrangements and plastic deformation into less dense, less mobile regions; there could also be a short range repulsion if the spot density gets too high, since grains will collapse into overly dilated regions. Such effects may be responsible for intermittent density waves in draining hoppers with narrow orifices [59, 60], and perhaps even shear banding in other amorphous materials, such as metallic glasses, with a different plastic yield criterion. However, we will see that the hypothesis of non-interacting spots already works rather well in cases of steady, dense granular flows.

\section{A simple model for steady flows}

Due to efficient dissipation by friction, granular materials subjected to steady forcing typically relax very quickly to a steady flowing state. For example, when a silo's orifice is opened, a wave of reduced density (spots) progates upward, leaving in its wake a nearly 
steady particle flow, which we associate with a steady flow of spots. This initial density wave can be seen very clearly in discrete-element simulations of various hoppersilo geometries [50]. For a narrow orifice, we have noted that intermittent flows with density waves can be observed [59, 60], but typical drainage flows are rather steady in time [49, 57]. Similarly, when a rough inner cylinder is set into uniform rotation in a Couette cell, shear dilation propagates outward, raising the level of the packing, until a steady flow profile is reached. We interpret this initial dilation as signaling the creation of spots on the cylinder, which quickly reach a steady distribution in the bulk.

Hereafter, we focus on describing steady flow profiles, with equilibrium spot densities. For simplicity, let us assume isotropic spot diffusion, $D^{\alpha \beta}=D_{2} \delta_{\alpha \beta}$, since fluctuations are dominated by the (largely isotropic) geometry of dense random packings. Using the spot size $L_{s}$ as the natural length scale, we can express the spot drift speed, $\left|\mathbf{D}_{1}\right|=L_{s} / \Delta t_{1}$, and diffusivity, $D_{2}=L_{s}^{2} / 2 \Delta t_{2}$, in terms of the times, $\Delta t_{1}$ and $\Delta t_{2}$, for drift and diffusion to reach this length.

The flow profile of a draining silo, normalized by the outflow speed, is approximately constant over a wide range of flow speeds, as has recently been verified to great precision in the experiments of Choi et al. [57]. Not only is the mean velocity profile independent of flow rate (over an order of magnitude in mean velocity), but fluctuations about the mean, such as vertical and horizontal diffusion and measures of "cage breaking", also depend only on the distance dropped, and not explicitly on time (or some measure of "granular temperature"). In statistical terms, changing the flow rate is like watching the same movie at a different speed, so that the random packing goes through a similar sequence of geometrical configurations regardless of the velocity. Similar features have also been observed in shearing experiments in Couette cells [61] and numerical simulations of planar shear 62 .

The experimental and simulational evidence, therefore, prompts the crucial approximation that $\Delta t_{1} / \Delta t_{2}=$ constant so as to uphold statistical invariance of the particle trajectories under changing the overall flow rate. This can be justified if spots perform random walks with displacements selected from a fixed distribution, set by the geometry of the random packing [27]. Here, we make the stronger assumption that the characteristic length of these random walks is the spot size $L_{s}$, so that $\Delta t_{1}=\Delta t_{2} \equiv \Delta t$. Our physical picture is that a spot represents a "cell" of localized fluidization (or plastic yield) of typical size $L_{s}$, which triggers further fluidization ahead of it and randomly propagates to a neighboring cell of similar extent. This picture is also consistent with the interpretation of $L_{s}$ as a velocity correlation length above.

With these hypotheses, the Fokker-Planck equation
(24) takes the simple time-independent form,

$$
\nabla \cdot\left(\hat{\mathbf{d}}_{s} \rho_{s}\right)=\frac{L_{s}}{2} \nabla^{2} \rho_{s}
$$

where $\hat{\mathbf{d}}_{s}(\mathbf{r})=\mathbf{D}_{1} /\left|\mathbf{D}_{1}\right|$ is the spot drift direction, determined by the mechanics of plastic yielding (below). The flow field is then

$$
\mathbf{u}=-\frac{L_{s}}{\Delta t} \int d \mathbf{r}^{\prime} w\left(\mathbf{r}, \mathbf{r}^{\prime}\right)\left(\hat{\mathbf{d}}_{s}\left(\mathbf{r}^{\prime}\right) \rho_{s}\left(\mathbf{r}^{\prime}\right)-\frac{L_{s}}{2} \nabla \rho_{s}\left(\mathbf{r}^{\prime}\right)\right)
$$

Equations (26) and (27) define a simplified Stochastic Flow Rule, with only one parameter, $L_{s}$, which need not be fitted to any flow profile. Instead, it can be measured independently as the velocity correlation length, which may be viewed as a dynamical material property.

\section{A mechanical theory of spot drift}

The main contribution of this paper is a simple theory connecting the spot drift direction to the stresses in MCP. The basic idea is to view the displacement of a spot as being due to a local event of material failure or fluidization. To make a quantitative prediction, we first define a cell of the material as the roughly diamond shaped region encompassed by two intersecting pairs of slip-lines, separated by $L_{s}$. When a spot passes through this cell, it fluidizes the material and thus locally changes the friction coefficient from the static value $\mu$ to the kinetic value $\mu_{k}$. This upsets the force balance on the cell and may cause a perturbative net force to occur.

The force diagram for a material cell occupied by a spot can be broken into the sum of two diagrams (Figure 12), one which is the static diagram multiplied by $\mu_{k} / \mu$ and one which contains only normal contact force contributions and a body force term. MCP requires the static diagram to be balanced, thus the latter is the only cause for a net force. A well-known corollary of the divergence theorem enables us to express the surface integral of normal contact stresses in terms of a gradient of $p$ giving

$$
\vec{F}_{n e t}=\left(1-\frac{\mu_{k}}{\mu}\right)\left(\vec{F}_{b o d y}-\cos ^{2} \phi \vec{\nabla} p\right)
$$

as an effective force which pulls on a cell as it is fluidized by a passing spot, causing the spot to preferentially drift in the opposite direction.

A spot cannot move in an arbitrary direction, however, since the material is at incipient yield only along the two slip lines. Therefore, the net force is constrained to pull the material cell along one of the slip-lines. The spot drift direction is then obtained by projecting (minus) the force, $-\vec{F}_{n e t}$, onto the slip-lines and averaging these two 
(a)

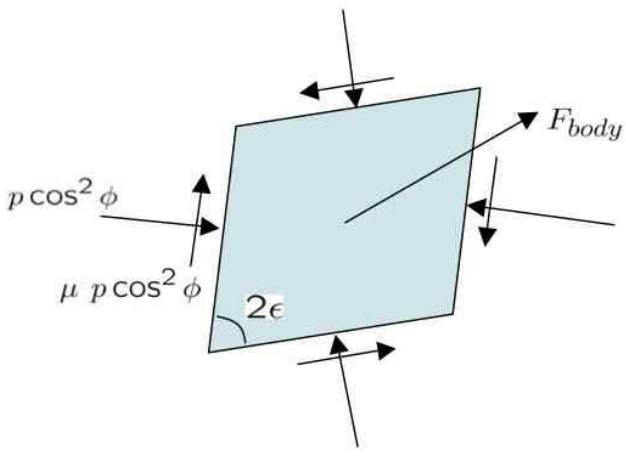

(b)

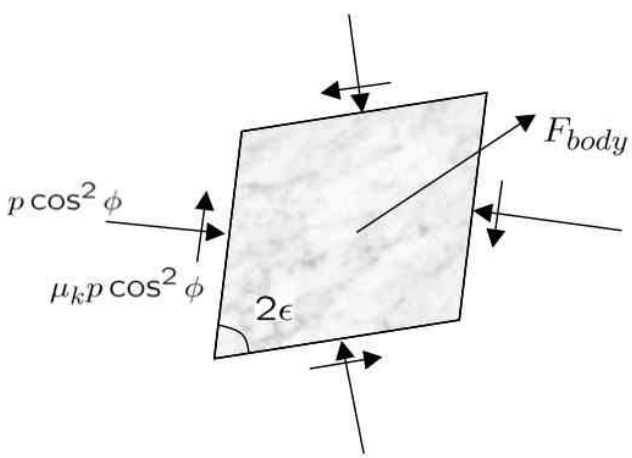

(c)
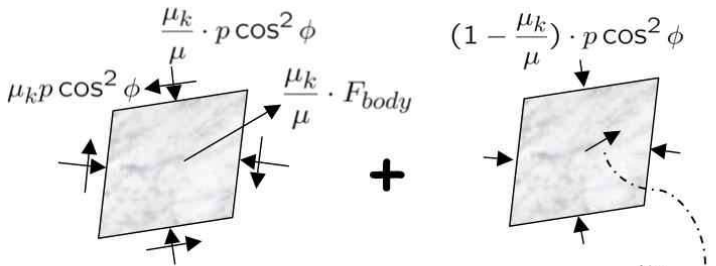

$\left(1-\frac{\mu_{k}}{\mu}\right) \cdot F_{b o d y}$

FIG. 12: (a) Material cell in static equilibrium.(b) A spot enters the cell fluidizing the material. In the force diagram, this means $\mu$ decreases to $\mu_{k}$. (c) The force diagram for the fluidized material cell is best analyzed by breaking it into the sum of two diagrams.

projection vectors with equal weight:

$$
\begin{aligned}
\vec{\xi}^{( \pm)} & =-\left(\vec{F}_{n e t} \cdot \hat{n}_{\psi \pm \epsilon}\right) \hat{n}_{\psi \pm \epsilon} \\
\hat{\mathbf{d}}_{s} & =\frac{\vec{\xi}^{(+)}+\vec{\xi}^{(-)}}{\left|\vec{\xi}^{(+)}+\vec{\xi}^{(-)}\right|}
\end{aligned}
$$

where $\hat{n}_{\theta}=(\cos \theta, \sin \theta)$. With a formula for $\hat{\mathbf{d}}_{s}$ now determined, the SFR as stated in equations (26) and (27) is now fully defined and ready for use.

This continuum mechanical theory of spot drift also helps us understand the sources of spot diffusion. As noted above and sketched in Figure 9] a material cell is a small fragment of a random packing, which is unlikely to be able to accomodate shear strain precisely along the slip-lines of the mean continuum stress field. Instead, the instantaneous slip-lines are effectively blurred by the discrete random packing. Still, we preserve the picture of spots moving along slip-lines in constructing $\hat{\mathbf{d}}_{s}$, but represent the additonal bluriness in the slip-line field by enforcing isotropic spot diffusivity.

\section{E. Frame indifference}

Finally, we must check that our flow rule satisfies frame indifference; solving for flow in different rigidly moving reference frames cannot give different answers in a fixed reference frame. Since the SFR is a $2 \mathrm{D}$ steady-state flow rule, the only flows we need to check for indifference are those with rotational/translational symmetry. In these cases, the particle velocity is a function of only one spatial variable and equation (26) for $\rho_{s}$ becomes a secondorder ODE. In solving the boundary value problem, we must ensure that grains along the walls have a velocity vector tangent to the walls. This constrains one of the two degrees of freedom in the set of possible solutions for $\rho_{s}$. Since (26) is homogeneous, the other degree of freedom must come out as a multiplicative undetermined constant. Thus the velocity profile is unique up to a multiplicative constant.

With only one constant, we cannot match boundary conditions for particle speed along more than one wall in general. So to solve for a flow between two walls, we must add rigid-body motions to the reference frame of the observer until we have the unique frame for which a solution exists matching both boundary conditions. This is an unexpected and welcome bonus of the SFR. Most flow rules in continuum mechanics enforce material frame indifference directly, i.e. the flow rule itself is derived to be automatically satisfied by any rigid-body motion, ensuring the same results independent of reference frame. Coaxiality achieves this by relating stress information only to strain-rate variables for instance. The SFR, however, upholds frame indifference indirectly in that the solution does not exist unless the problem is solved in exactly one "correct" frame of reference.

We have thus integrated the spot concept with the theory of plastic stresses treating spots as the "carriers of plasticity". We note that up to our granular-specific determination of the drift direction, the SFR principle can be applied to any amorphous isotropic material with a small characteristic length scale (dominant randomness) and a yield criterion.

\section{APPLICATIONS TO GRANULAR FLOW}

The Stochastic Flow Rule is quite general and in principle can be applied to any limit-state plasticity model of stresses, with different choices of the yield function to describe different materials. In this section, we apply the simplest SFR (26)-(27) to granular materials with MCP 
stresses and compare its predictions to a wide range of existing experimental data for steady dense flows. In calculating stresses, we assume a typical friction angle of $\phi=30^{\circ}$. It is known that for spherical grains, the friction angle usually lies in a somewhat narrow range of about $20^{\circ}-30^{\circ}$ and can be as large as $50^{\circ}$ for some anisotropic, highly angular materials 1]. In the examples we consider, however, varying the $\phi$ value in this range has very little macroscopic effect in our model.

The spot size $L_{s}$ has a much larger effect, so we focus on its role in a variety of dense flows. We emphasize that we do not fit $L_{s}$ to any flow profile below. Instead, we simply use the range $L_{s}=3-5 d$ for dense flowing sphere packings inferred independently from spatial velocity correlations in silo drainage experiments [57] and simulations [28] (see Fig. 11). This is consistent with our view of the correlation length, $L_{s}$, as a fundamental geometrical property of a flowing granular material.

Without any fitting parameters, we will apply the simple SFR to several prototypical flows. Each has different forcing and symmetries and, to our knowledge, they cannot be simultaneously described by any existing model. Our first example is granular drainage to a small orifice in a wide flat-bottomed silo, driven entirely by gravity. Our second example is shear flow in an annular Couette cell driven by a moving rough inner cylinder, where gravity plays no role. Our third example is the dragging of a loaded plate over a semi-infinite material at rest, which combines gravitational forces and boundary forcing. Lastly we apply the SFR to a canonical freesurface flow, the continuous avalanching of a granular heap. The transition to a rapidly flowing surface shear layer on a heap will also lead us into a discussion of how rate-dependent effects, such as Bagnold rheology, may naturally extend into our model.

Throughout our treatment of the various examples, the first step will be to solve (26) to obtain the "unconvolved" velocity field

$$
\mathbf{u}^{*}=-L_{s} \hat{\mathbf{d}}_{s} \rho_{s}+\frac{L_{s}^{2}}{2} \nabla \rho_{s}
$$

which corresponds to the SFR velocity (27) for a pointlike influence function $w=\delta\left(\left|\mathbf{r}-\mathbf{r}^{\prime}\right|\right)$. For the most part, $\mathbf{u}^{*}$ is the "skeleton" for the full solution $\mathbf{u}$ because convolving $\mathbf{u}^{*}$ with a general spot influence merely blurs out the sharper features of $\mathbf{u}^{*}$. In some situations with wide shear zones, such as silo flow, the convolution has only a minor effect, but in others with narrow shear bands, at the scale of the spot size, the convolution step is essential for self-consistency and accuracy.

\section{A. Silos}

The flow profile in a flat-bottom silo geometry is wellknown for its striking similarity to the fundamental solution of the diffusion equation. As noted above, early models of silo flow explained this based on the upward diffusion of voids from the orifice [24, 25, 56]. Without reference to a specific microscopic mechanism, Nedderman and Tüzun later derived the same equations based on a continuum constitutive law [1, 26]. They asserted that the horizontal velocity component $u$ is proportional to the horizontal gradient of the downward velocity component $v$,

$$
u=b \frac{\partial v}{\partial x}
$$

since particles should drift from regions of slow, dense flow toward regions of faster, less dense (more dilated) flow. Assuming small density fluctuations, mass conservation applied to the $2 \mathrm{D}$ velocity field, $\mathbf{u}=(u,-v)$ then yields the diffusion equation for the downward velocity,

$$
\frac{\partial v}{\partial z}=b \frac{\partial^{2} v}{\partial x^{2}}
$$

where the vertical direction $z$ acts like "time". The diffusivity $b$ is thus really a "diffusion length", to be determined empirically. An advantage of the continuum formulation is that it avoids the paradox (resolved by the Spot Model [27]) that the classical picture of void random walks requires $b \ll d$, while experiments invariably show $b>d$.

Solving the Kinematic Model in the wide flat-bottomed silo geometry with a point orifice gives the familiar Green function for the diffusion equation,

$$
v(x, z)=\frac{e^{-x^{2} / 4 b z}}{\sqrt{4 \pi b z}} .
$$

This gives an excellent match to experimental data close to the orifice (e.g. see Figure [13), although the fit gradually gets worse with increasing height, as the flow becomes somewhat more plug-like. Nevertheless, Gaussian fits of experimental data have provided similar estimates of $b=1.3 d$ [57], $1.3-2.3 d$ [49], $2.3 d$ [26], 3.5d [48], and $2 d-4 d$ [54] for a variety of granular materials composed of monodisperse spheres.

We now apply our theory to this flow geometry and see how it connects to the Kinematic Model. Applying equation (30) using the stress field described by equations (22) and (23) gives uniform upward spot drift; $\vec{F}_{n e t}$ comes out as pointing uniformly downward and the slip-lines are symmetric about the vertical (see Figure 14). The SFR (26) then reduces to

$$
\frac{\partial \rho_{s}}{\partial z}=\frac{L_{s}}{2}\left(\frac{\partial^{2} \rho_{s}}{\partial x^{2}}+\frac{\partial^{2} \rho_{s}}{\partial z^{2}}\right)
$$

although we emphasize that this form applies only when the walls are smooth or equivalently when the silo width is large. The last term, which represents vertical diffusion of spots (relative to the mean upward drift), makes this equation for the spot density differ somewhat from the simple diffusion equation for the downward velocity 

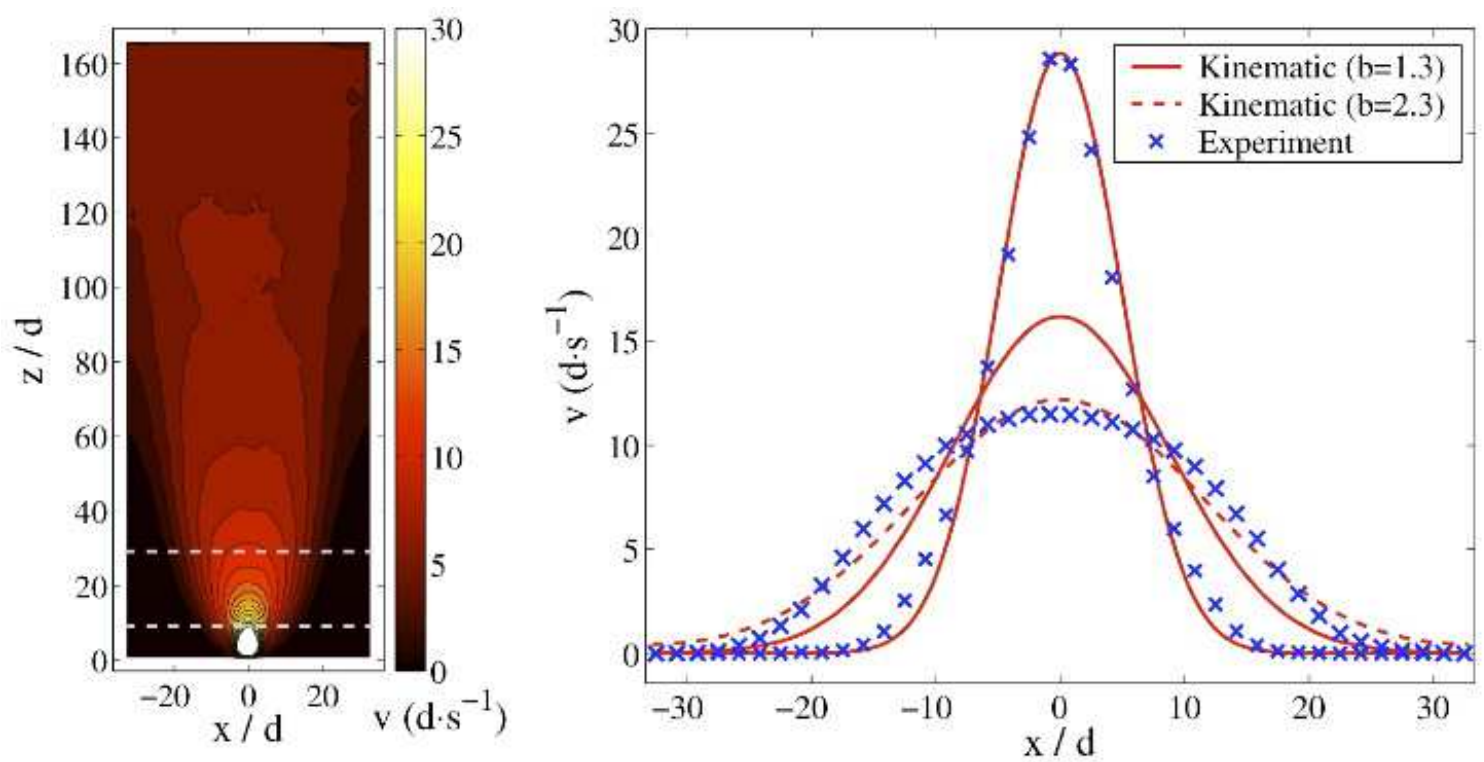

FIG. 13: The mean velocity profile in a wide quasi-2d silo of $3 \mathrm{~mm}$ glass beads from Ref. 49]. Horizontal slices of the downward velocity component near the orifice, indicated in the complete flow profile on the left, are shown on the right, and compared to the predictions of the Kinematic Model with two choices of the parameter $b$. The Stochastic Flow Rule for MCP for a wide silo (without side walls) gives a similar velocity field with $b \approx 1.5-2.5 d$.

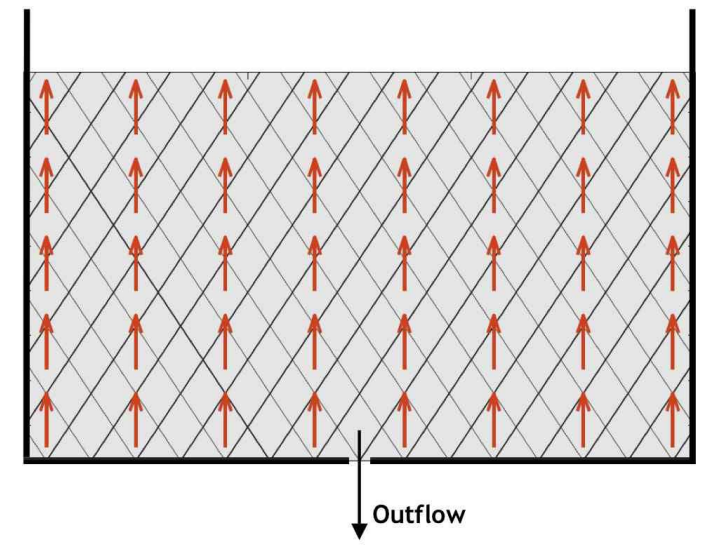

FIG. 14: The flat-bottomed silo geometry. The intersecting black lines represent the slip-line field as determined from solving the stress balance equations of MCP, and the red vector field is the spot drift direction, as determined from the SFR. In this highly symmetric geometry, the spot drift precisely opposes the gravitational body force, $\hat{d}=-\hat{g}$.

of the Kinematic Model. Consistent with our model, vertical diffusion, with a similar (but not identical) diffusion length as horizontal diffusion, has been observed in recent silo-drainage experiments [49, 57].

The general solution of (35) can be expressed as a Fourier integral,

$$
\rho_{s}=\frac{1}{2 \pi} \int_{-\infty}^{\infty} e^{i k x} A(k) e^{\frac{z}{L_{s}}\left(1-\sqrt{1+L_{s}^{2} k^{2}}\right)} d k
$$

where $A(k)$ is the Fourier transform of the spot density at the bottom $(z=0)$. The narrowest possible orifice allowing for flow is the case of a point source of spots, $\rho_{s}(x, 0) \propto \delta(x), A(k) \propto 1$ (which is also the Green function). Convolution with a spot influence function of width $L_{s}$ produces a downward velocity profile on the orifice of width $L_{s}$. Unlike the Kinematic Model (or any other continuum model which does not account for the finite grain size), our theory thus predicts that flow cannot occur unless the orifice is at least as wide as one spot, $L_{s}=3-5 d$.

The details of flow very close to the orifice, $z=O\left(L_{s}\right)$, are controlled by the choice of boundary condition, reflecting the dynamics of dilation, contact-breaking, and acceleration at the orifice, which are not described by our bulk dense-flow model. Rather than speculate on the form of this boundary condition, we focus on the bulk region slightly farther from the orifice. For $z \gg L_{s}$ (and $L_{s} k \ll 1$ ), the vertical diffusion term becomes unimportant, and the Green function tends to a Gaussian

$$
v(x, z) \sim \frac{e^{-x^{2} / 2 \sigma_{v}^{2}(z)}}{\sqrt{2 \pi \sigma_{v}^{2}(z)}}
$$

where the variance is

$$
\sigma_{v}^{2}(z) \sim L_{s} z+O\left(L_{s}^{2}\right)
$$

(The second term is an offset from convolution with the spot influence function, which also depends on the choice of boundary conditions.)

There has been no prior theoretical prediction of the kinematic parameter $b$, which we interpret as the spot 
diffusion length 27]. Comparing (34) and (37), we obtain $b=L_{s} / 2=1.5-2.5 d$ without any fitting, beyond the independent determination of $L_{s}$ from velocity correlations. This prediction is in excellent agreement with the experimental measurements listed above. However, the model does not predict the apparent increase of $b$ with height, as the flow becomes more plug like. This may be due to the breakdown of the assumption of incipient yield higher in the silo, where the shear is greatly reduced, and it may require modeling stresses more generally with elasto-plasticity.

In any case, we are not aware of any other model of silo flow with a plausible basis in mechanics. It is noteworthy that we assume active silo stresses (driven by gravity), as typically assumed in a quasi-static silo. As a result, we do not require a sudden switch to passive stresses (driven by the side walls) upon flow initiation, as in existing plasticity theories based on the Coaxial Flow Rule [1]. Our use of the standard MCP model for stresses in quasi-static silos also suggests that the SFR may predict reasonable dependences on the geometry of the silo or hopper, wall roughness, and other mechanical parameters. In contrast, the Kinematic Model fails to incorporate any mechanics, and, not surprisingly, fails to describe flows in different silo/hopper geometries in experiments [49]. Testing our model in the same way would be an interesting direction for future work, since it has essentially no adjustable parameters.

\section{B. Couette cells}

The key benefit of our model is versatility; we will now take exactly the same model, which is able to describe wide silo flows driven by gravity, and apply it to TaylorCouette shear flows in annular cells driven by a moving boundary. The granular material is confined between vertical rough-walled concentric cylinders and set into motion by rotating the inner cylinder. The flow field has been characterized extensively in experiments and simulations, and several theories have been proposed for this particular geometry [19, 20, 33, 63]. For example, a good fit of experimental data for Couette cells can be obtained by postulating a density and temperature dependent viscosity in a fluid mechanical theory [20], but it is not clear that the same model can describe any other geometries, such as silos, hoppers, or other shear flows.

To solve for the MCP stresses in the annular Couette geometry, we first convert the stress balance equations to polar coordinates $(r, \theta)$ and require that $p$ and $\psi$ obey radial symmetry. This gives the following pair of ODEs:

$$
\begin{aligned}
\frac{\partial \psi^{*}}{\partial r} & =-\frac{\sin 2 \psi^{*}}{r\left(\cos 2 \psi^{*}+\sin \phi\right)} \\
\frac{\partial \eta}{\partial r} & =-\frac{2 \sin \phi}{r\left(\cos 2 \psi^{*}+\sin \phi\right)}
\end{aligned}
$$

where $\eta=\log p$ and $\psi^{*}=\psi+\frac{\pi}{2}-\theta$. Although $\psi^{*}$ has an implicit analytical solution, $\eta$ does not, so we solve

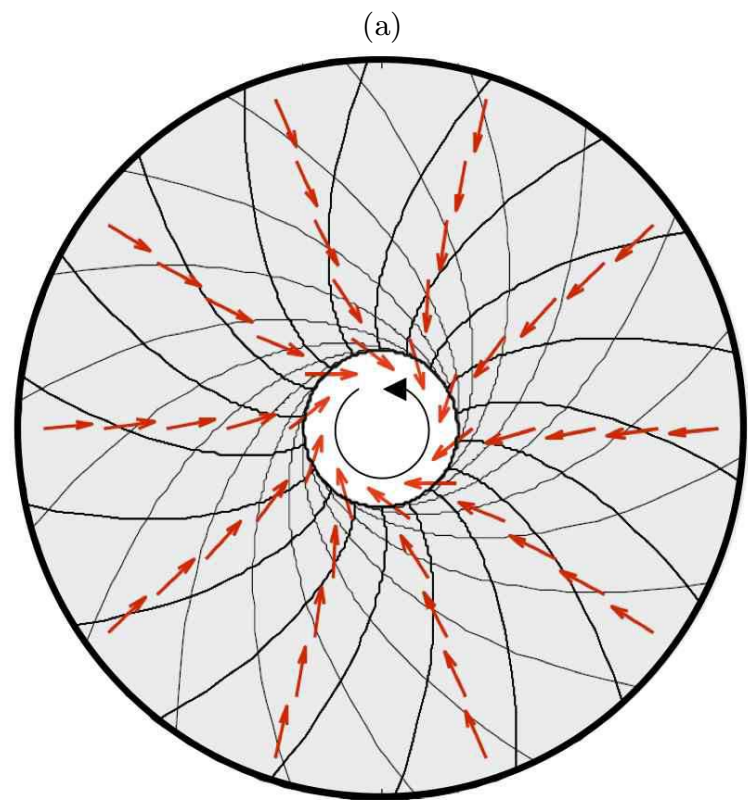

(b)

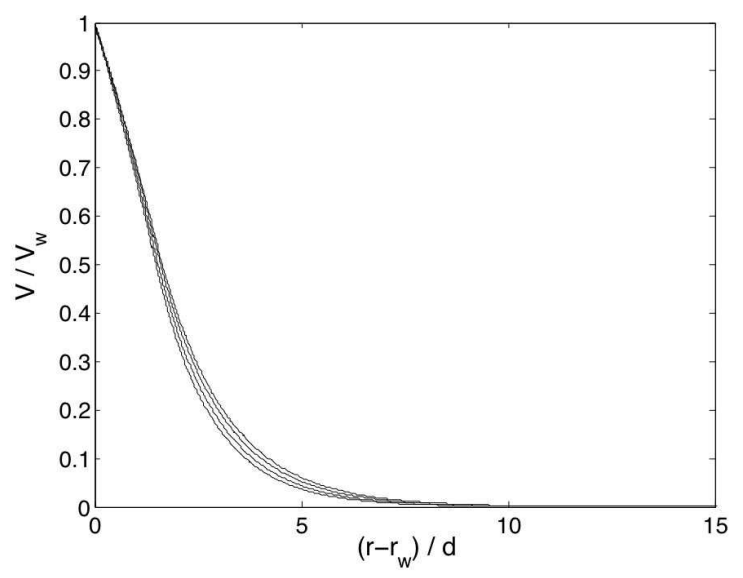

FIG. 15: (a) A plan view of the annular Couette cell geometry, where the granular material is confined between concentric vertical cylinders. The rough wall is rotated anti-clockwise while the outer wall is held fixed. The crossing black lines within the material are the slip-lines as found from MCP, and the red vector field is the spot drift as determined by the SFR. (b) Normalized SFR velocity from as a function of distance from the inner wall with inner cylinder radius $15 \mathrm{~d}$, $25 \mathrm{~d}, 50 \mathrm{~d}$, and $100 \mathrm{~d}$ (from bottom to top curves, respectively). The friction angle is $\phi=30^{\circ}$, and the spot size is $L_{s}=3 d$.

these equations numerically using fully rough inner wall boundary conditions $\psi^{*}\left(r_{w}\right)=\frac{\pi}{2}-\epsilon$ and any arbitrary value for $\eta\left(r_{w}\right)$. The resulting slip-lines are shown in Figure 15(a).

In the Couette geometry, the average normal stress, $p$, decreases with radial distance, which implies that the fluidization force on material, $\vec{F}_{n e t}$, is everywhere directed outward. We then apply equation (30) to calculate the drift direction $\hat{\mathbf{d}}_{s}(r)$ by projecting the vector $\vec{F}_{n e t}$ onto 
slip-lines, and implement the SFR, exploiting symmetry which allows only a nonzero velocity in the $\hat{\theta}$ direction. This implies

$$
\mathbf{u}^{*} \cdot \hat{r}=0=-L_{s}\left(\hat{\mathbf{d}}_{s} \cdot \hat{r}\right) \rho_{s}+\frac{L_{s}^{2}}{2} \frac{\partial \rho_{s}}{\partial r}
$$

which yields a solution for $\rho_{s}$ up to a scalar factor. We then use $\rho_{s}$ to calculate the $\theta$ component of the (unconvolved) velocity once again using the SFR equation,

$$
\mathbf{u}^{*} \cdot \hat{\theta}=-L_{s}\left(\hat{\mathbf{d}}_{s} \cdot \hat{\theta}\right) \rho_{s} .
$$

It turns out, as we may have expected, that $\mathbf{u}^{*}$ has a shear band at the inner wall with nearly exact exponential decay. The length scale of this decay is the spot size, $L_{s}$, since this is the velocity correlation length, beyond which the inadmissible shear at the inner cylinder can be effectively dissipated by the material.

The thinness of the shear band requires that, for consistency, we must take into account the finite spot size in reconstructing the velocity field through the convolution integral (27). For simplicity we will use a uniform spot influence function, i.e.

$$
w(\mathbf{r})=\frac{4}{\pi L_{s}^{2}} H\left(\frac{L_{s}}{2}-|\mathbf{r}|\right)
$$

where $H(x)$ is the Heaviside step function. To evaluate the integral (27), we also must make a hypothesis about how spots operate when they overlap one of the walls. Random packing dynamics near walls is different than in the bulk and sensitive to surface roughness, and further detailed analysis of experiments and simulations will be required to elucidate the collective mechanism(s). Here, the precise shape of spots near the wall has little effect, except to flatten out the spike in velocity that occurs near the wall in the unconvolved velocity $\mathbf{u}^{*}$. As simple first approximation, used hereafter in this paper, we will view the space beyond each boundary as containing a bath of non-diffusive spots at uniform concentration whose flux is such that the particle velocity invoked "inside" the boundaries directly mimics the rigid motion of the walls. This effectively "folds" part of the spot influence back into the granular material when it overlaps with the wall. The resulting velocity field is shown in Figure 15(b), where normalized velocity is shown versus distance from the inner cylinder wall for $L_{s}=3 d$ for a wide range of inner cylinder radii.

The predicted flow field - without any fitting - is in striking agreement with a large set of data from experimental and discrete-element simulations for different cylinder radii and grain sizes $19,33,63,65]$. As shown in Figure [16] the experimental data compiled by GDR Midi 33] falls almost entirely within the predicted SFR velocity profiles, by setting the spot size to the same typical range of correlation lengths, $L_{s}=3-5 d$, measured independently in a quasi-2D silo (Figure 11). Viewing the data on a semilog plot shows that the agreement extends all the way into the tail of the velocity distribution. We emphasize that the same simple theory, with (a)

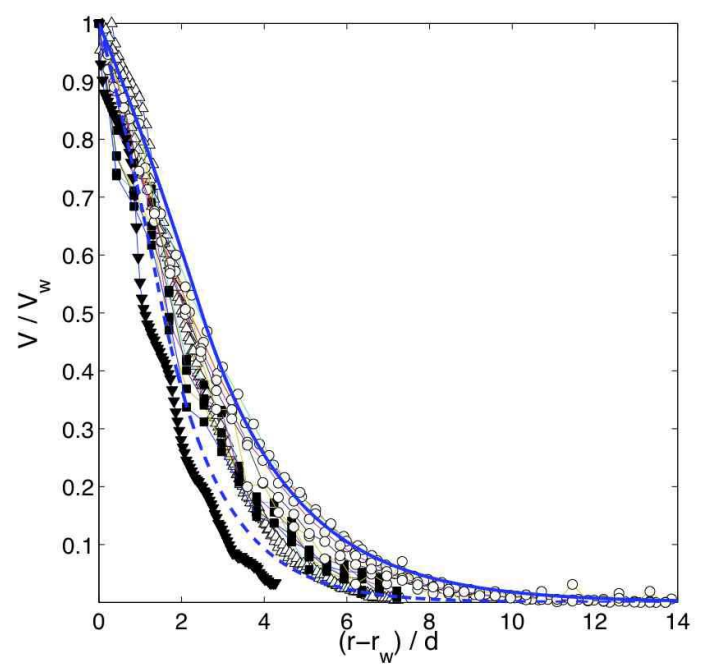

(b)

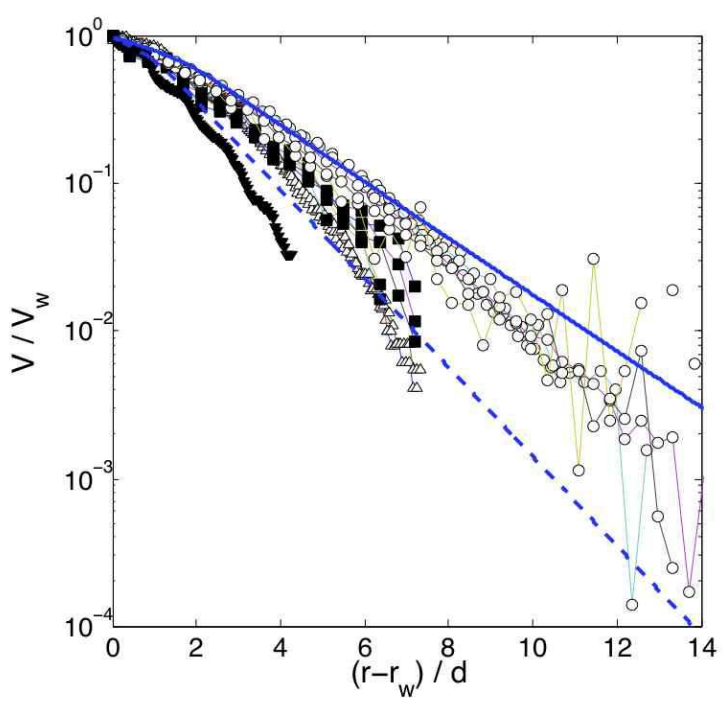

FIG. 16: Theory versus experiment for the normalized velocity in annular Couette cells on (a) linear and (b) semilog plots. The dashed curve is the predicted SFR velocity field with $L_{s}=3 d$, while the solid line is for $L_{s}=5 d$; both curves are for an inner cylinder radius of $r_{w}=80 d$ and $\phi=30^{\circ}$. Experimental measurements (points) for a wide range of inner and outer cylinder radii are shown from the compilation of data shown in Figure 3c of [33]. (The experimental data is courtesy of GDR Midi and originates from the work of [64], [65], 66], and [67].)

the same range of $L_{s}$ values, also accurately predicts silo flows above, as well as other situations below. Unifying all of this data in a single simple theory without any empirical fitting constitutes a stringent quantitative test.

It is interesting to note the behavior close to the wall, especially in thin Couette cells. In experiments [33], annular flow profiles are known to have a Gaussian correction term when the thickness of the cell becomes non- 


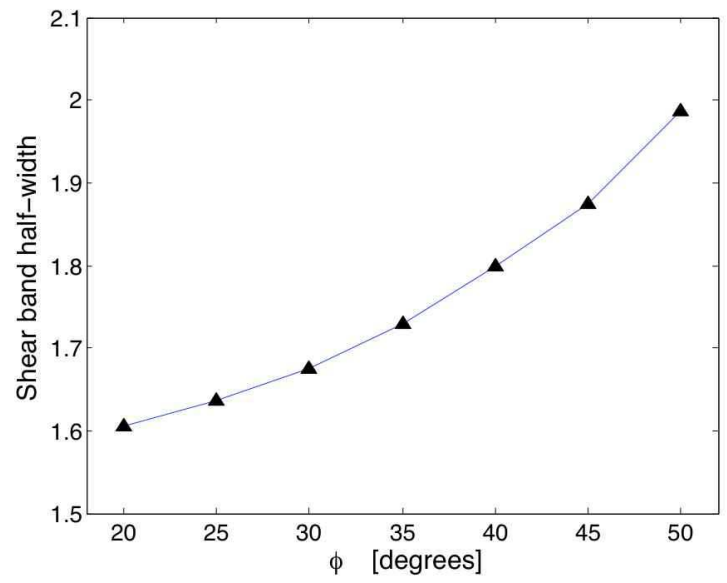

FIG. 17: Predicted variation in the width of the shear band with SFR over the standard range of granular friction angles for the annular Couette geometry. $\left(L_{s}=3 d, r_{w}=80 d\right)$

negligible in terms of particle size. This slight flattening near the wall is apparent in our model as well and is a byproduct of convolving with the spot influence. We thus interpret this feature as another sign of the strongly correlated motion of particles, primarily with the "cage" of nearest neighbors, as approximately described by the spot mechanism. In this calculation, we used a uniform spot influence, but have noticed relatively little sensitivity of the predicted flow profile, for different strongly localized influence functions, such as a Gaussian, $w \propto e^{-2 r^{2} / L_{s}^{2}}$. A detailed comparison of the model to experimental data may provide fundamental insights into the spot influence, and thus the collective dynamics of random packings, near a rough wall at the discrete particle level.

The experimental results shown in Figure 16 come from apparati with inner wall radii ranging from $14 d-100 d$. The relatively small variations in the data sets over such a large range of inner radii clearly indicates that the inner wall radius is not a crucial length scale in the flow. The plotted theoretical prediction uses an inner radius of $\approx 80 d$, but, as can be seen in Figure 15(b), our results depend only minimally on the inner cylinder radius. Indeed, the meso-scale correlation length of $L_{s}=3-5 d$ is the dominant length scale in our theory for this geometry.

To substantiate an earlier claim, we now consider how the friction angle $\phi$ affects the flow properties (holding $L_{s}$ fixed) according to our model. We can see this most clearly by observing how the shear band half-width (i.e. the distance from the wall to the location where velocity is half-maximum) varies over the $\phi$ range for usual granular materials $\left(\approx 20^{\circ}-50^{\circ}\right)$. As shown in Figure 17 the half-width changes by $<0.4 d$ over the entire range and by $<0.1 d$ for the range of laboratory-style spherical grains. This very weak influence of internal friction agrees with simulations in the Couette geometry by Schöllmann 68 .

\section{Plate dragging}

We now examine perhaps the simplest situation where gravity affects the shear band caused by a moving rough wall. Consider slowly dragging a rough plate horizontally across the upper surface of a deep (semi-infinite) granular material. The plate maintains full contact by pressing down on the surface with pressure $p_{0} \cos ^{2} \phi$. The profile of the shear band which forms below the plate depends on the relative loading pressure, $q_{0}=p_{0} / f_{g}$, where $f_{g}$ is the weight (gravitational body force) density of the material.

The plate-dragging flow field can be found using a procedure analagous to the annular Couette cell, but enforcing horizontal instead of radial symmetry. With $y$ measuring distance below the plate, the stress balance equations give

$$
\begin{aligned}
\psi_{y} & =\frac{-\sin 2 \psi}{2 q(\cos 2 \psi-\sin \phi)} \\
q_{y} & =\frac{\cos 2 \psi}{\cos 2 \psi-\sin \phi}
\end{aligned}
$$

where $q(y)=p(y) / f_{g}$ is the average normal stress scaled to the weight density. The fluidization force will push material downward and spots upward resulting in a flow profile that decays close to exponentially near the moving wall.

Experiments 70, 71 and simulations 72, 73, 74] offer differing assessments of the details of the flow profile away from the shear band, but the dominant exponential decay behavior is clearly observed in all. The displayed SFR prediction (Figure 18) uses loading parameters from Tsai and Gollub 69 in order to appropriately compare with their results. Although the general properties of the flow appear to be represented well by the model, we do notice that the predicted range of typical flows is too small to fully encompass the experimental data. There could be a number of reasons for this discrepancy, but it is worth pointing out that the quasi-2D plate-dragging geometry is rather difficult to realize in experiments. For example, this experiment was executed by rotating a loaded washer-type object on top of an annular channel, and it was observed that the sidewalls pushing in the third dimension actually did play some role.

In Refs. 73 and 75], simulations of this environment indicate that the shear band width increases with increasing loading of the top plate. As can be seen in Figure [19] our theory captures this general trend of increasing loading causing increasing shear band width. However, the swing in band size predicted by our theory is not large enough to match the range of band sizes in simulations [3] and 75] in which the shear band half-width can be as large as several tens of particle diameters for large enough $q_{0}$ and diverges as $q_{0} \rightarrow \infty$ (i.e. zero gravity). In cases such as these where the value of $q_{0}$ becomes very large, as we will discuss in more depth after the next section, we believe a new phenomenon begins to dominate our meso-scale argument and that this phenomenon 
(a)

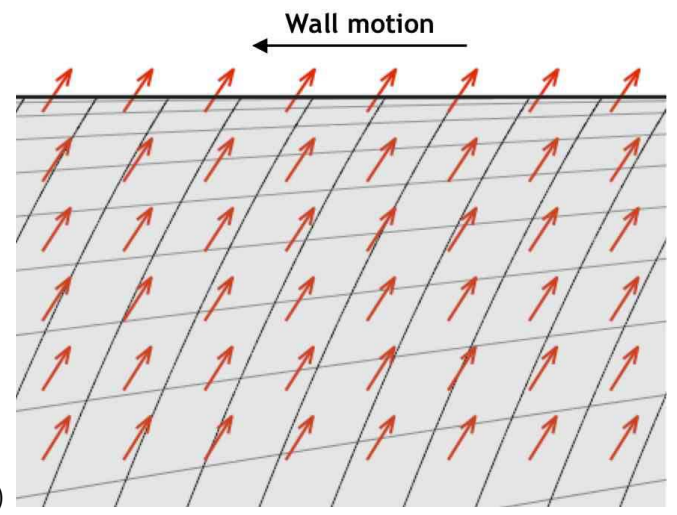

(b)
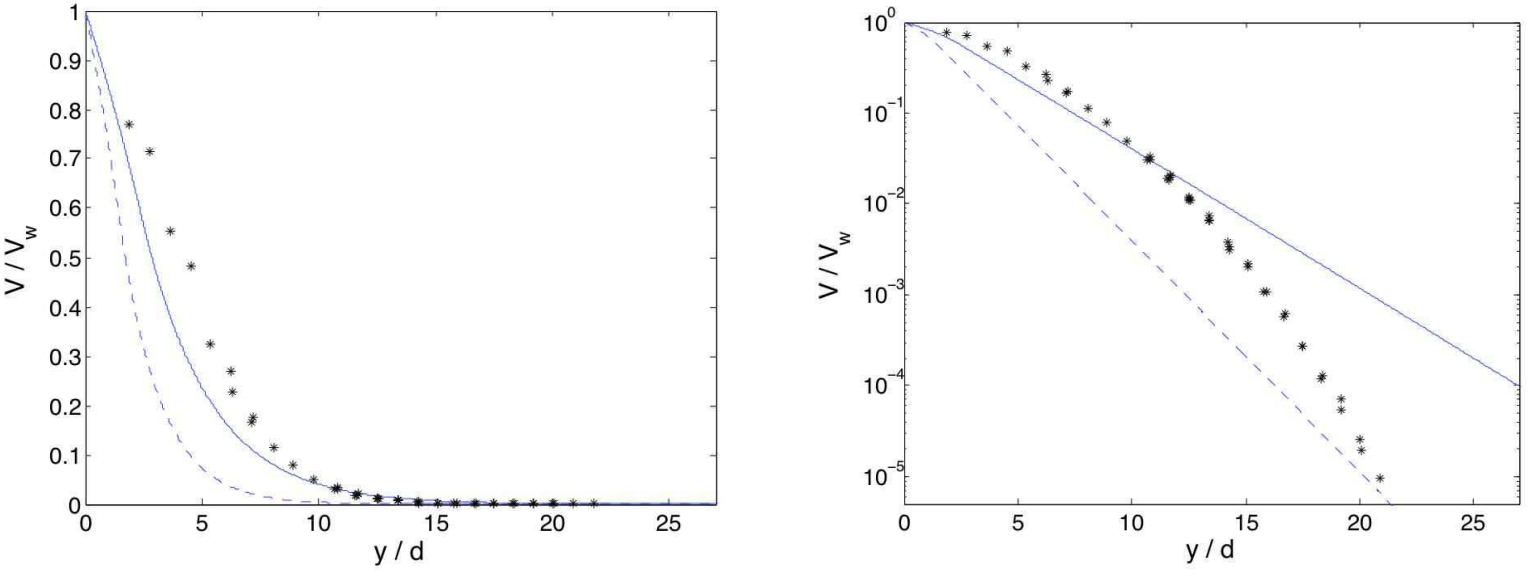

FIG. 18: (a) The plate-dragging geometry. The top wall drags along the top of a bed of granular material. The crossing black lines within the material are the slip-lines as found from MCP, and the vector field is the spot drift as determined by the SFR. (b) Theory against experiment for the plate dragging geometry. Theory: $\mathrm{L}=3(---), \mathrm{L}=5$ (-). Experiment (*) courtesy of the authors of 69$]$.

may be attributed to a particular property of the slip-line field.

\section{Slow heap flows}

We now examine a prototypical free surface flow. Very close to the respose angle, a granular heap which is slowly but consistently re-fed grains undergoes a particular type of motion characterized by avalanching at the top surface and a slower "creeping" motion beneath. This type of flow has been studied in experiments [6] and simulations [77]. Though heap flows with faster top shear layers have also been studied [33, 78] we will focus for now on the slower regime, which more closely resembles a quasi-static flow where the SFR might apply.

This kind of flow is stable, but indeed quite "delicate" in the sense that relatively small changes to the system parameters (i.e. flow rate, height of the flowing layer) can invoke large changes to the qualitative flow profile especially in the top layers [7]. We will describe and attempt to explain this effect more in the next section.

The heap geometry is depicted in Figure 20 along with the corresponding spot drift field and slip-line field. Any gravity driven free surface flow problem for which the stresses and flow are approximately invariant in the direction parallel to the surface will have limit-state stresses that obey the following relations:

$$
\begin{aligned}
\psi & =-\epsilon \\
p & =\frac{f_{g} y}{\cos \phi}
\end{aligned}
$$

where $y$ is the depth measured orthogonally from the free surface. Note that in limit-state theory, for selfconsistency, the static angle of repose is identical to the internal friction angle $\phi$, which is a reasonable assertion but still debated in the community. (By "static repose angle" we refer to the angle of inclination below which a flowing system jams; in simulations of flow down a rough inclined plane, it has been shown that this angle does vary in a narrow range depending on the height of the flow [77, 79].) Applying equation (30) to these equations gives a simple expression for the spot drift vector:

$$
\hat{\mathbf{d}}_{s}=\frac{\left(1+\sin ^{2} \phi,-\sin \phi \cos \phi\right)}{\left|\left(1+\sin ^{2} \phi,-\sin \phi \cos \phi\right)\right|}
$$




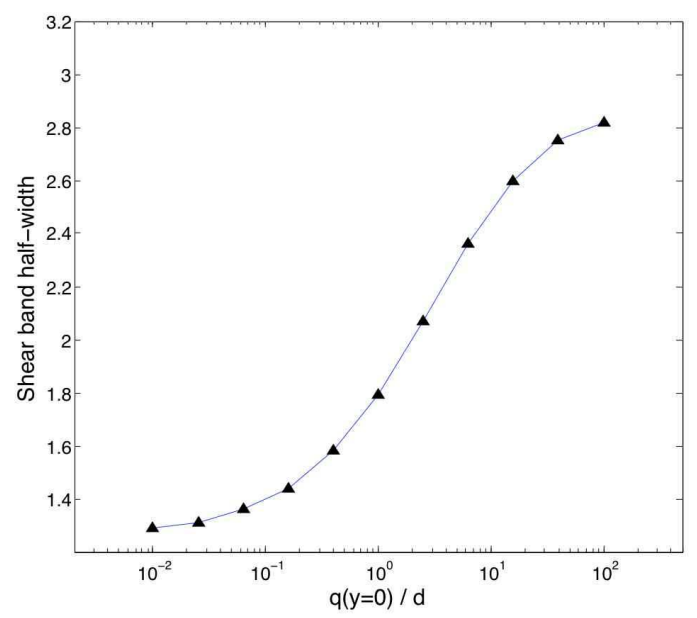

FIG. 19: Plot of theoretical shear band half-width vs relative loading pressure $q_{0}=p(y=0) / f_{g}$ of the top plate (where $f_{g}$ is the weight density). The calculation assumes $L_{s}=5 d$

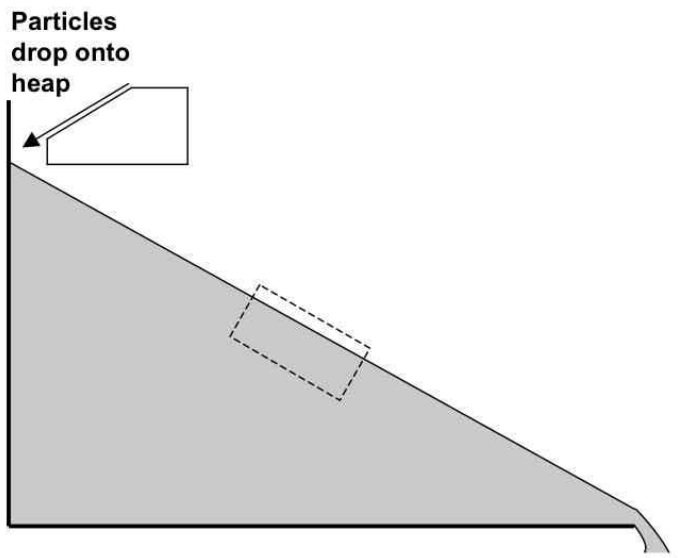

(a)

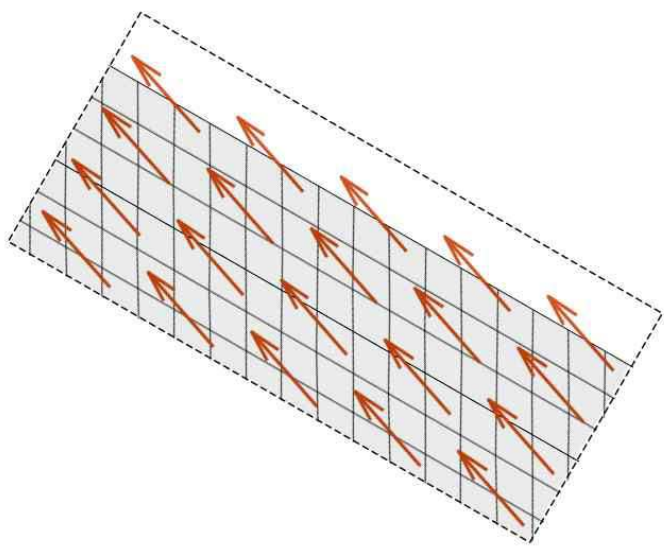

FIG. 20: (a) The heap flow setup. (b) The dashed rectangle in (a) is enlarged; the slip-line field from MCP is plotted along with the drift field from the SFR.
We may then apply the SFR, which simplifies upon requiring that the flow run parallel to the free surface (i.e. $\left.\mathbf{u}^{*}=(-u, 0)\right)$.

Since the drift field is uniform, we obtain an analytical solution for the unconvolved velocity:

$$
u=u(0) \exp \left(\frac{-y \sin 2 \phi}{L_{s}} \sqrt{\frac{2}{5-3 \cos 2 \phi}}\right)
$$

Thus our model predicts that the velocity decays exponentially off the free surface. In cases like these, where the boundary of the flow makes no contact with a rigid wall, it is less clear how the spots (and free volume) might behave near the flowing free surface. To avoid addressing this issue in detail, we neglect convolution with the spot influence function and simply assume $\mathbf{u} \approx \mathbf{u}^{*}$.

In their experiments on slow heap flow, Lemieux and Durian [6] have shown that the velocity profile in the flowing top layers is indeed well approximated by an exponential decay. Furthermore, they found the flow in this regime to be continuous and stable. The decay law they obtained is

$$
u / u(0) \approx \exp \left(-\frac{y}{4.5 d}\right)
$$

which is very close to our predicted solution for $L_{s}=3 d$ :

$$
u / u(0)=\exp \left(-\frac{y}{4.58 d}\right)
$$

Silbert et. al 77] report finding a similar decay profile at low flow rates in simulations of flow down a rough inclined plane, although the avalanching at the surface was intermittent. In conclusion, we have demonstrated a fourth, qualitatively different situation where the same simple MCP/SFR theory predicts the flow profile, without adjusting any parameters.

\section{TRANSITION FROM THE SFR TO BAGNOLD RHEOLOGY}

\section{A. Breakdown of the SFR}

In the last two examples, plate dragging and slow heap flow, there are limits where the SFR fails to predict the experimental flow profiles. In this section, we will explain why the breakdown of the SFR is to be expected in these cases and others, whenever slip-lines approach "admissibility" and coincide with shear planes. In this singular limit of the SFR, we postulate a transition to Bagnold rheology. The stochastic spot-based mechanism for plastic yielding is thus replaced by a different physical mechanism, the free sliding along shear planes.

For example, consider the case of plate dragging above. At large relative loading, the flow field resembles that of a zero-gravity horizontal shear cell (between shearing flat plates), and it appears that the SFR breaks down: With body forces and $\nabla p$ both going to 0 , equation (30) 
gives $\vec{F}_{n e t}=\mathbf{0}$ implying that spots have no drift and consequently the only SFR solution is $\mathbf{u}=\mathbf{0}$.

Problems also occur with flow down a rough inclined plane: Slightly increasing the flow rate (and consequently the flow height) or inclination angle causes the velocity vs depth relationship to exit the exponential decay regime detailed above and undergo significant changes, passing first through a regime of linear dependence [80, 81] to a regime resembling a $3 / 2$ power-law of depth 79,82 , 83, 84] opposite in concavity to the exponential decay regime.

Why does the velocity profile for inclined plane flow undergo many different qualitative regimes depending delicately on system parameters, while others (e.g. silos, annular cells) appear to be only weakly affected and almost always exhibit the same (normalized) velocity profile? Tall inclined plane flows and zero-gravity planar shear flows have been successfully described in multiple experiments and simulations [62, 79, 82, 83, 85] by the empirical scaling law of Bagnold [16]. In this section, we suggest a means to reconcile and perhaps eventually combine these theories into a coherent whole.

\section{B. Bagnold rheology}

Let us briefly review Bagnold's classical theory of granular shear flow. In its original form, "Bagnold scaling" expresses a particular rate-dependency for granular flow whenever the solid fraction is uniform throughout:

$$
\tau \propto \dot{\gamma}^{2}
$$

where $\dot{\gamma}$ is the rate of simple shear. To account as well for static stresses arising from the internal friction, a related variant of this scaling law is commonly used [86]:

$$
\tau-\mu \sigma \propto \dot{\gamma}^{2}
$$

It is in some sense a law for how the yield criterion can be exceeded when non-negligible strain-rates can absorb the extra shear stress. This constitutive law alone is an incomplete flow theory since it provides no way of predicting whether or not the solid fraction will be uniform during flow or how a non-uniform solid fraction affects the above rheology. Bagnold originally explained the quadratic relationship between stress and strain-rate in terms of binary collisions as the joint effect of both the particle collision rate and the momentum loss per collision being directly proportional to the strain-rate [16]. Despite this collision-based argument, however, Bagnold scaling has been observed to hold well into the dense regime, whenever the solid fraction is approximately constant throughout the system. This seemingly contradictory observation can be justified in the hard-sphere limit (without body forces) by a Newtonian invariance argument [62], although it calls into question the underlying physical mechanism.

Zero-gravity planar shear flow and thick inclined plane flow both exhibit nearly uniform density and thus have been employed as test cases for Bagnold scaling. In the planar-shear environment, the shear and normal stresses acting on the shear planes are spatially constant throughout the flow. Equation (49) therefore implies that the strain-rate is uniform; as a result, the velocity varies linearly from one wall to the other. This result is known as Uniform Shear Flow (USF) and is easily verified in simulations of Lees-Edwards boundary conditions. For example, the rheology (49) has been demonstrated in the simulations of 85 .

Applying Bagnold scaling to the inclined plane geometry, slightly above static repose, gives a shear stress excess which grows linearly with depth and thus a shearing rate that grows as the square root of depth. This implies a velocity profile of the form

$$
u \propto h^{3 / 2}-y^{3 / 2}
$$

for $y$ the depth variable and $h$ the height of the flowing material (with no-slip bottom boundary condition). In this way, Bagnold scaling successfully explains the $3 / 2$ power law dependence noted above.

\section{Slip-line admissibility}

The seemingly disparate flow mechanisms of the SFR and Bagnold rheology can be reconciled very naturally by considering the geometry of the slip-lines. In plasticity theory, all flows can be classified based on "slip-line admissibility". For admissible slip-lines, boundary conditions are such that the flow can, and presumably does, take place by continuous shearing along only one family of slip-lines. In mathematical terms, the slip-lines are admissible for a given flow, whenever the double-shearing continuum flow-rule (14) allows multiple solutions to the boundary value problem.

Slip-line admissibility is the exception, not the rule, since it is highly unlikely that the prescribed velocity boundary conditions are fulfilled by a continuous shear on either slip-line family. Be that as it may, it so happens that planar shear flow and inclined plane flow are both slip-line admissible. This special property is shared by no other flow geometry studied in this paper, or, to our knowledge, elsewhere in the granular materials community. (Contrast the slip lines in Figure 21 with those in Figures 14 and 15)

There is also an interesting difference in the density distributions. For admissible flows, the volume fraction is nearly uniform, and Bagnold rheology has a reasonable physical justification. For the more common case of inadmissible flows, as in silos and Couette cells, the volume fraction is typically highly nonuniform. In such cases, the SFR seems to provide an excellent description of the flow, and Bagnold rheology clearly does not apply.

These observations motivate us to think of admissibility as a criterion for two very different microscopic mechanisms for granular flow: In admissible flows, material motion is a viscous dragging of material "slabs" 
(a)

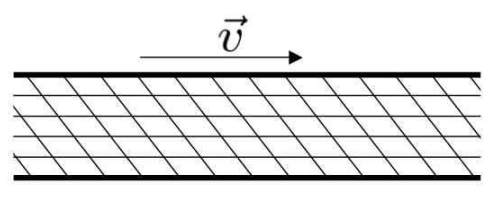

(b)

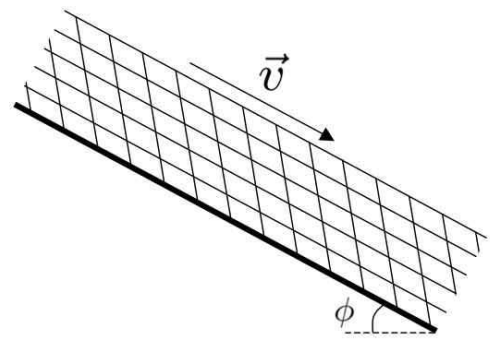

FIG. 21: (a) Slip-line field for gravity-free planar shear flow, (b) Slip-line field for inclined plane flow. Note that in both cases the shear planes will be aligned completely with one slip-line family.

along one slip-line family (Bagnold dominated), whereas in inadmissible flows there is no clear choice as to which slip-line family should control the motion and thus material randomly chooses between both slip-line families (SFR dominated). Perhaps admissibility in the slip-line field causes the solid fraction to remain roughly uniform because the material in no sense has to collide head-on into neighboring material for it to move. In flows where non-uniform dilation does occur, experiments have shown the motion is nearly independent of any local Bagnold rheology, encouraging our strong distinction between the dynamics of flow problems of differing admissibility status 65,87 .

These considerations all lead us to the fundamental conjecture:

Slip-line admissibility is a geometrical and mechanical indicator as to the relative importance of rate-dependency (Bagnold rheology) over rate-independency (SFR) in a dense granular flow.

This means that a flow which has an admissible limitstate stress field will be dominated by rate-dependent effects when the yield criterion is only slightly exceeded.

\section{Redistribution of excess shear stress}

A more rigoruous physical justification of our conjecture can be made utilizing limit-state stresses and observing the effect of pushing the system above yield. Bagnold rheology is a statement connecting the shear stress excess (i.e. amount by which $\tau$ exceeds $\mu \sigma$ ) along a shear plane to the rate of simple shear along the plane. We must emphasize that shear planes and slip-lines are not equivalent terms; slip-lines are defined by the quasi-static stresses as lines along which $\tau-\mu \sigma=0$, whereas shear planes are defined entirely by the velocity profile. In an admissible system, the shear planes coincide with one slip-line family. In inadmissible systems, the shear planes almost everywhere do not coincide with slip-lines.

With admissible slip-lines, excess shear stress tends to be uniformly distributed throughout the system, resulting in global Bagnold rheology. For example, consider a zero-gravity planar shear cell. If we were to apply additional shear stress to the body in a manner aligned with the admissible slip-line family, e.g. by increasing the wall shear stress above yield by some amount $\Delta \tau$, that additional shear stress would distribute itself within the material precisely along the slip-lines. Every horizontal slip-line within the bulk would thus receive a boost in shear stress of size $\Delta \tau$. In limit-state theory the sliplines have the highest possible $\tau-\mu \sigma$ value a quasi-static material element can take - zero. Adding $\Delta \tau$ additonal shear stress to the slip-lines means that $\tau$ will maximally exceed $\mu \sigma$ precisely along the slip-lines, and, by admissibility, every shearing plane. As a result, there will be a Bagnold contribution everywhere. Similarly, if we took a limit-state inclined plane geometry and increased the tilt angle some amount, an analagous boost in shear stress along the admissible slip-line family would occur causing $\tau$ to exceed $\mu \sigma$ precisely along all the shear planes.

In contrast, with inadmissible slip-lines, excess shear stress tends to remain localized where it is applied, and the SFR dominates the rest of the flow. For example, consider annular Couette flow. As can be seen in Figure 15. the slip-lines only coincide with the shear planes (which in this case are concentric circles) along the inner wall of the cell. If the inner wall were given an increase $\Delta \tau$ in applied shear stress, torque balancing requires the shear stress along any concentric circle within the material to receive a boost of $\Delta \tau \cdot r_{w} / r$. Suppose the shear cell has inner wall radius $40 d$ and the boost in wall shear is significant, say $\Delta \tau=\tau_{w} / 10$. Solving for $\tau-\mu \sigma$ along the shear planes in this situation gives a very different result than in the previous case - here $\tau$ will only exceed $\mu \sigma$ along the shear planes that are less than $1.4 d$ off the inner wall. So, regardless of whether the density is or is not uniform, Bagnold scaling would at best only apply in an almost negligibly thin region near the wall. If the wall friction were less than fully rough, this region would further decrease.

\section{E. A simple composite theory}

The preceding discussion indicates that, in general, one can use the admissibility status of the system to choose whether or not the flow should obey the SFR or Bagnold rheology, or perhaps some combination of the two. Indeed, it seems reasonable that when slip-lines are $a p$ proaching admissibility (e.g. plate-dragging with high $q_{0}$ ) or when an admissible system is only slightly pushed 
above yield (e.g. inclined plane flow near static repose) we must account for contributions from both effects simultaneously. It is beyond the scope of this paper to postulate the precise microscopic dynamics (and derive corresponding continuum equations) for this regime, but we can at least give a sense of how the more general theory might look.

In general, we envision a smooth transition from rateindependent SFR dynamics to rate-dependent Bagnold dynamics controlled by the distribution of shear stress excess. This implies the coexistence of (at least) two different microscopic mechanisms: SFR and admissible shear. The SFR contribution would derive from the usual spot-based, quasi-static stochastic dynamics; the Bagnold contribution would come from a rate-dependent shearing motion along the appropriate slip-line family whenever there is a small excess shear stress (beyond the limit state) applied on a boundary which causes shear stress excess along the shear planes within.

The two mechanisms should have different statistical signatures. For shear deformation along admissible sliplines, we would expect anisotropic velocity correlations. In the direction perpendicular to the shear plane, the correlation length should be somewhat shorter than the typical spot size, since slip-line admissibility allows flow to occur with less drastic local rearrangements, farther from jamming. In the directions parallel to the shear plane, however the correlation length could be longer, since material slabs sliding along shear planes may develop more rigid, planar regions. It would certainly be interesting to study velocity correlations in heap flows at different inclinations and plate-dragging experiments under different loads to shed more light on the microscopic mechanisms involved in the SFR to Bagnold transition.

For the remainder of this section, we make a first attempt at a composite model, simply a linear superposition of SFR and Bagnold velocity fields:

$$
\mathbf{u}=\alpha \mathbf{u}_{\mathrm{SFR}}+\beta \mathbf{u}_{\mathrm{Bag}} .
$$

which could have its microscopic basis in a random competition between the two mechanisms, when slip-lines are near admissibility. Here, $\mathbf{u}_{S F R}$ is an SFR solution for the flow, using the limit-state stress field everywhere, and $\mathbf{u}_{\text {Bag }}$ is obtained from the excess shear stress on a boundary by integrating the Bagnold strain rate $\dot{\gamma}=\sqrt{\tau-\mu \sigma}$ over those shear planes for which $\tau-\mu \sigma$ has been boosted above zero. (Note that we ignore the condition of uniform density for Bagnold rheology since we conjecture that uniform density is actually a geometric consequence of slip-line admissibility and will arise naturally whenever Bagnold rheology dominates the flow.)

A reasonable first approximation is that the SFR and Bagnold solutions individually fulfill the necessary boundary conditions for the velocity profile since, under the right circumstances, either can be made to dominate the other. The constant $\beta$ is the Bagnold proportionality constant which may depend on the density of the flow among other parameters [85]. Since the SFR is a rate- independent flow model, $\mathbf{u}_{S F R}$ can always be multiplied by a positive constant (observe that equation (26) is homogeneous in $\rho_{s}$ ), and thus we allow the scalar multiple $\alpha$. Given some determinable form for $\beta, \alpha$ is chosen such that $\mathbf{u}$ fits the velocity boundary conditions. This seems reasonable for moving walls (as in plate dragging), but not for free surfaces, whose boundary velocity should also be predicted by the theory. In such cases, where $\alpha$ is not clearly defined in this simple model, one could use other empirical relations, such as the Pouliquen Flow Rule for inclined plane flows 82], to deduce the free boundary velocity, and thus $\alpha$.

\section{F. Some applications of the composite theory}

Using our very simple composite theory, we will now revisit a few geometries that were troublesome for the SFR alone. Extending the theory with a smooth transition to Bagnold scaling controlled by slip-line admissibility seems to resolve the experimental puzzles and capture the basic physics of granular shear flows. In the cases we consider below, we do not change the value of $\alpha$ as we increase the shear stress excess; this way the relative importance of Bagnold effects are easier to isolate.

\section{Planar shear cell}

In a zero-gravity planar shear cell, $\mathbf{u}_{S F R}=0$, but the Bagnold solution for any amount of shear stress excess is of the form $\mathbf{u}_{B a g}=k y$, and thus the composite solution, regardless of the values $\alpha$ and $\beta$, is a homogeneous flow between the two rough plates. The lack of a "background" SFR solution in this case may explain why Bagnold rheology is almost exactly observed in simulations of this geometry over a wide range of strain rates 62 .

\section{Rough inclined plane}

For flow down a rough inclined plane, the SFR solution is an exponential decay (47), and the Bagnold solution is a $3 / 2$ power law (50). When the material is only slightly above static repose, a shear stress excess along the shear planes will exist but will be very small; it goes as $\sqrt{\Delta \theta}$ for an incline $\Delta \theta$ above static repose 86 . As a result, $\mathbf{u}_{B a g}$ will be small in magnitude, and the SFR solution will show through as the "creeping flow" with exponential decay. As $\Delta \theta$ increases, the increased shear stress excess will cause the Bagnold contribution to increase, and the flow will eventually morph into the $3 / 2$ power law dependence of pure Bagnold scaling. In between, where both contributions are of similar magnitude, the superposition of the two flow fields gives a predicted profile that appears approximately linear, since the SFR and Bagnold solutions are of opposite concavity. Thus, the composite SFR-Bagnold formulation appears to be able 


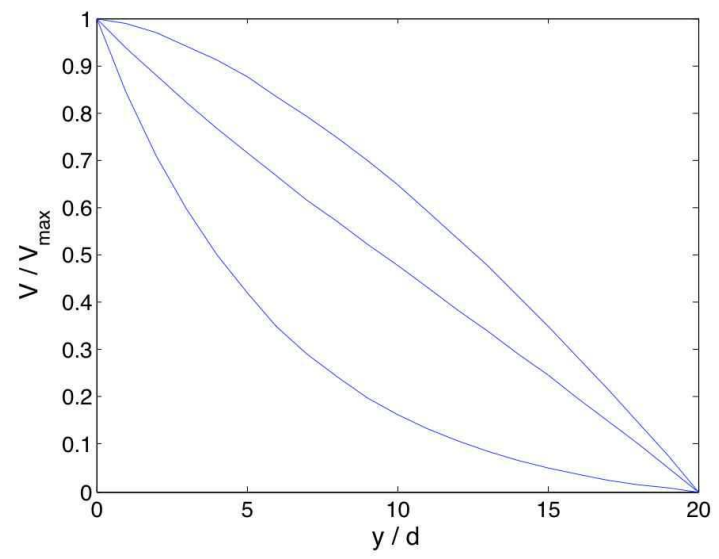

FIG. 22: Some predicted velocity profiles for flow down a rough inclined plane as a function of depth. Note that all three known flow behaviors; exp decay, linear, 3/2 power law; appear in proper relationship to the inclination (see Ref. [7]). (Bottom) Incline near static repose, fully SFR dominated $\left(L_{s}=4 d\right)$; (Middle) Increased inclination angle, Bagnold to SFR ratio of 3:1; (Top) Further increase to inclination; fully Bagnold dominated.

to explain the various flow regimes in inclined plane flow, which have been observed in experiments and simulations (see Figure 22).

Recent experimental work of Pouliquen [88] seems to support this analysis; it is found that inclined plane flow occurring at lower inclination angles exhibits spatial velocity correlations near the typical spot size (as the SFR would imply), but as inclination increases, the correlation length appears to decrease, an effect we might attribute to an increased dominance of Bagnold scaling (a phenomenon not goverened by a correlation length) over the SFR.

\section{Rapid heap flows}

The composite theory also seems consistent with rapid heap flows. When the flow rate down the heap increases, the region near the surface resembles inclined plane flow in any one of its various flow regimes, whereas the region beneath the surface flow undergoes creep motion which decays close to exponentially [33, 78] (see Figure 23). We can justify this in terms of slip-line admissibility: The slip-lines throughout the system (see Figure 21) have the same form in both regions. In the surface region, the sliplines are admissible because there is nothing blocking the motion from being a simple shearing along the slip-lines. In the creep region, however, the gate (or the ground) prevents global shearing along the slip-lines and thus the slip-lines are inadmissible and the SFR dominates.

We can equivalently explain heap flow in terms of shear stress excess. The excess incurred by increasing the heap angle will distribute itself differently in the two regions.

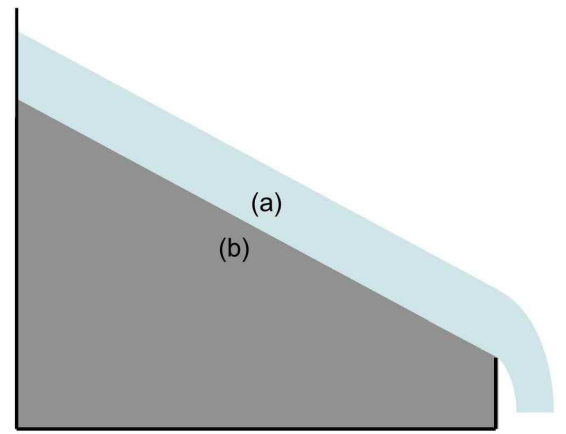

FIG. 23: Standard heap flows enable one to see both the SFR and Bagnold contributions spearately in one flow geometry. (a) The surface region is dominated by Bagnold scaling. (b) The creep-region beneath adheres to the SFR.
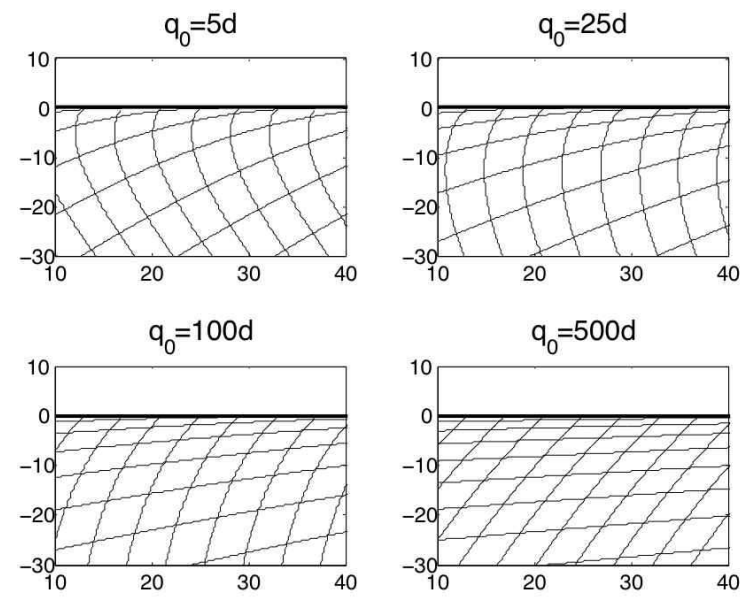

FIG. 24: Plate-dragging slip-lines approaching admissibility as $q_{0}$ increases.

In the surface region, the excess can only be absorbed along the shear planes by inducing a strong Bagnold dependence. However, the gate at the bottom of the heap will support any shear stress excess on the creep region. (Note that the slip-lines in the creep region all hit the gate, or the ground.) Thus the full flow will be the sum of an exponentially decaying SFR solution superposed with a significant Bagnold-type solution which starts at the surface and cuts off at the interface with the creep zone.

\section{Plate dragging under a heavy load}

We will now explain the comment made at the end of the plate-dragging section. The slip-lines of a platedragging geometry can be pushed drastically close to full admissibility by simply increasing the relative loading of the top plate, $q_{0}$, above a certain non-excessive amount 
(see Figure 24). To see the effects of approaching admissibility more carefully, say we take a limit-state platedragging setup and pull the plate slightly harder, inducing a super-yield shear stress boost of $\Delta \tau$ under the plate. Bagnold effects should appear wherever, as a consequence of stress balancing, a shear stress excess results along a shear plane. The shear planes are horizontal lines in this case, and at limit-state, the stresses along any horizontal obey

$$
\tau-\mu \sigma=-\mu f_{g} y=-\mu \frac{p_{0}}{q_{0}} y
$$

When the extra shear stress is applied, a shear stress excess of $\Delta \tau-\mu p_{0} y / q_{0}$ will form for $0 \leq y \leq \frac{\Delta \tau}{\mu p_{0}} q_{0}$. Accordingly the Bagnold flow contribution will have a shear zone whose depth extends into the granular bed as an increasing linear function of $q_{0}$ for a fixed shear stress boost $\Delta \tau$ and fixed downward plate-pressure (i.e. we decrease the material weight density to increase $q_{0}$ ). Integrating the Bagnold shear rate gives that the relative size of the Bagnold contribution should also increase with increasing $q_{0}$. With $q_{0}$ large enough, therefore, the SFR contribution will be dwarfed by a Bagnold term with a larger shear band. As $q_{0} \rightarrow \infty$ the slip-lines become completely admissible and the shear band width diverges as we would expect.

The mismatch in shear band size between the data of Tsai and Gollub and the SFR could be due, among other possible reasons, to the fact that $q_{0} \approx 380 d$ was large enough to make the Bagnold contributution sizeable. This could also explain the large shear bands found in 73]. A detailed comparison of experiments and simulations with different versions of a composite SFR/Bagnold theory would be an interested direction for future work.

\section{CONCLUSION}

\section{A. Highlights of the present work}

We have proposed a stochastic flow rule (SFR) for granular materials, assuming limit-state stresses from Mohr-Coulomb plasticity (MCP). In the usual case where slip-lines are inadmissible (inconsistent with boundary conditions), we postulate that flow occurs in response to diffusing "spots" of local fluidization, which perform random walks along slip-lines, biased by stress imbalances. The spot-based SFR corrects many shortcomings of classical MCP and allows some of the first reasonable flow profiles to be derived from limit-state stresses, which engineers have used for centuries to described the statics of granular materials.

Our theory notably differs from all prior continuum theories (cited in the introduction) in that it is derived systematically from a microscopic statistical model [27]. The Spot Model is already known to produce realistic flowing random packings [28], and what we have done is to provide a general mechanical theory of spot dynamics. Evidence for spots has been consistently found in spatial velocity correlations in simulations 28] and experiments 57] (Fig. 11) on silo drainage.

Beyond its fundamental physical appeal, the SFR seems to have unprecedented versatility in describing different granular flows. It has only two parameters, the friction angle $\phi$ and correlation length (spot size) $L_{s}$, which are not fitted; they are considered properties of the material which can be measured independently from flow profiles. For monodisperse frictional spheres, the SFR can predict a variety of different flows using the same spatial velocity correlation length, $L_{s} \approx 3-5 d$, measured in experiments and simulations. This is perhaps the most compelling evidence in favor of the spot mechanism which underlies the SFR.

We have shown that the SFR can describe a rather diverse set of experimental data on granular flows. Some flows are driven by body forces (silo and heap flows); others have body forces, but are driven by applied shear (plate-dragging); still others are driven by applied shear without body forces (annular shear flow). Some geometries have straight boundaries (silos, heaps, platedragging), and yet the theory works equally well for highly curved boundaries (annular shear flow). Some of the flows exhibit shear localization (annular shear flow, plate-dragging, heap flow), and yet the theory correctly predicts wide shear zones in silo flow. It is noteworthy that the same, simple model, correctly predicts and places shear bands in geometries where they arise for very different reasons - gravity causes the shear band in platedragging, and yet the geometry (through the $\nabla p$ term in the drift) causes the shear band in annular Couette flow. We are not aware of any other theory (including classical MCP) which can quantitatively describe more than one of these flows, let alone without empirically fitting the velocity profiles.

\section{B. Comparison with partial fluidization}

It is interesting to compare our approach to the continuum theory of partial fluidization of Aranson and Tsimring [31, 32]. Although it lacks any microscopic basis, their theory also introduces a diffusing scalar field to control the dynamics, as opposed to a classical stress/strainrate relation. The analog of our spot density is the "order parameter" $\rho$, which measures the degree of "fluidization" of the continuum by mixing two different types of stresses, corresponding to distinct "liquid" $(\rho=0)$ and "solid" $(\rho=1)$ phases. Given the stress tensor for the material in a static solid state, $\sigma_{i j}^{0}$, the stresses in a flowing granular material are modeled by adding some degree of viscous stresses, as in a Newtonian liquid:

$$
\sigma_{i j}=\left(\rho+(1-\rho) \delta_{i j}\right) \sigma_{i j}^{0}+\eta \dot{E}_{i j}
$$

where $\eta$ is the viscosity. The order parameter controlling the balance of these two stress tensors is postulated to 
obey a reaction-diffusion equation,

$$
(\Delta t) \frac{\partial \rho}{\partial t}=l^{2} \nabla^{2} \rho+\rho(1-\rho)(\rho-\delta)
$$

for collision time $\Delta t$, grain length scale $l$, and a function $\delta$ of the stress state, which is greater than 1 where the material is above the static yield criterion, less than 0 where below the dynamic yield criterion, and between 0 and 1 otherwise. One benefit of this model is that it can be used for unsteady flows. In principle, the SFR may also describe time-dependence through the spot FokkerPlanck equation (24), but we have only developed and tested the theory so far for steady flows, starting from (26).

For the sake of comparison, consider a steady flow modeled by partial fluidization and the SFR. The difference is that the spot equation (26) couples diffusion to a drift depending on frictional yielding, whereas the order parameter equation (54) balances diffusion with a nonlinear source term, ressembling a chemical reaction rate, which indirectly mimicks the effect of a Coulomb yield criterion. Interestingly, if the SFR could be extended to an elasto-plastic model without making the incipient failure assumption (see below), a similar nonlinear source term may have to be added to the spot equation to account for the need to destroy spots when they enter zones below yield. It is also notable that our argument for why a spot drifts, i.e. a localized stick-slip type of shear stress decrease along the spot boundary, is reminiscent of equation (53) wherein the shear stress goes down in the presence of fluidization. In this sense, a higher spot concentration in our model is similar to a higher degree of partial fluidization.

The similarities, however, seem to end there. One difficulty with the partial fluidization approach is that it cannot easily describe rate-independent effects since the motion stems from a viscous form in the stress tensor. Also in sharp contrast to our approach based on plasticity, partial fluidization does not provide a clear theory of the static solid stresses in the limit of no flow, opting instead to deal with environments for which the open components of this tensor are not needed (simple shear flows). This could perhaps be modified, but if the theory is to be fully general, equation (53) would need a frame-independent form where foreknowledge of the shear planes is not necessary to properly apply fluidization. These considerations as well as selecting boundary conditions on the order parameter, seem to be the primary limitations in testing partial fluidization in more general situations, such as those considered here with the SFR.

\section{Future directions}

In spite of some successes, we still do not have a complete theory of dense granular flow. There are at least
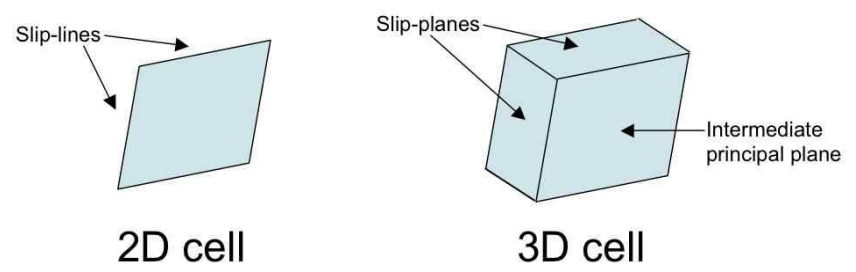

FIG. 25: The difference between 2D and 3D continuum cells which could be used to construct the SFR.

three basic limitations of the SFR, about which we can only offer some preliminary ideas to guide future work.

\section{Slip-line inadmissibility}

Although most slip-line fields are inadmissible, the SFR breaks down as slip-lines approach admissibility. We have already begun to extend the model into this regime by conjecturing that slip-line admissibility is associated with Bagnold rheology, as excess shear stress (above the limit-state) drives a local shear rate along the most admissible slip-lines. We have shown that a simple linear superposition of Bagnold and SFR flow fields with appropriate boundary conditions can describe a variety of composite flows, exhibiting both Bagnold and SFR behavior in different limits or segregated into different regions. These include planar zero-gravity shear, various inclined-plane and heap flows, and plate-dragging at large loading. However, more work is needed to develop and test a composite SFR/Bagnold theory, both at the continuum level and in terms of the two microscopic mechanisms.

\section{2D symmetry}

Through MCP, the SFR is currently used only in quasi-2D geometries. In efforts to extend the theory to $3 \mathrm{D}$, a good test case would be the split-bottom Couette cell, which displays a wide, diffusive shear band [55], reminscent of a draining silo. The $2 \mathrm{D}$ limitation may not be so difficult to overcome, although any plasticity theory is more complicated in three dimensions, than in two. As usual, constructing a 3D limit-state stress field requires an additional hypothesis to close the stress equations. In $3 \mathrm{D}$, a general material point at incipient failure with distinct principal stresses $\sigma_{1}>\sigma_{2}>\sigma_{3}$ is intersected by a pair of slip planes angled $2 \epsilon$ apart. We cannot therefore encase a 3D cell of material within slip planes as we are able to do to a $2 \mathrm{D}$ cell with slip-lines. However, the principal plane on which $\sigma_{2}$ acts, the intermediate principal plane, can be used along with the slip-planes to encase a 3D material cell. This is legitimate because, if such a cell underwent slip-plane fluidization, the net material force 
would be guaranteed to point parallel to the intermediate principal plane; since the intermediate principal plane offers no shear resistance, the material can slide along this plane, while simultaneously sliding along a slip-plane.

To apply the SFR then, the drift vector should still be calculated from equations (28) and (30), but all vectors must be projected first into the $\sigma_{1} \sigma_{3}$-plane since the $\sigma_{2}$ direction is not involved in slip-plane fluidization. The shape of a spot and its diffusivity would likely be anisotropic, with different values in the intermediate direction, since the main source of diffusion is slip-plane fluidization.

If ever the intermediate principal stress equals either the major or minor principal stress, as in the Har Von Karman hypothesis, incipient failure is upheld on a cone instead of intersecting slip-planes. When this degenerate case occurs, the material cell can be encased serveral different ways depending on the surrounding stress states. This must be determined before we can rigorously define how to apply fluidization and the SFR.

\section{Incipient yield everywhere}

The SFR assumes stresses near a limit state. While incipient yield is believed to be a good hypothesis in many situations, even during dense flow (which should be checked further in DEM simulations), it clearly breaks down in some cases, at least in certain regions. This is perhaps the most difficult limitation to overcome, since the limit-state assumption is needed to fully determine the stress tensor. Without it, the material effectively enters a different state most likely governed by some nonlinear elastic stress strain law which is far more difficult to apply.

We have already argued that such a transition away from incipient yield must exist in some granular flows, due to the strong tendency of granular materials to compactify into a rigid solid state when shaken (e.g. by nearby flowing regions) but not sufficiently sheared. A good example is a tall narrow silo with smooth side walls, where the SFR holds near the orifice, but breaks down in the upper region, ressembling a vertical chute. The broad shear band localizes on the side walls, as a rigid central plug develops, which likely falls below incipient yield.

A more robust elasto-plastic theory for the stress state would relax our limit-state constraints and allow for material to fall below the yield criterion where it is described by elasticity. The SFR could then be applied only where the material is at yield and everywhere else the material does not deform plastically. Elasto-plasticity theory operates just as well in $3 \mathrm{D}$ as in $2 \mathrm{D}$ which is a key benefit over limit-state plasticity. However, our model as we have already presented it is far simpler than elasto-plasticity and yet still manages accurate results when applied to limit-state stress fields.
As the SFR matures as a theory of granular flow, it would also be interesting to apply it to other amorphous materials, such as metallic glasses, and to develop new simulation methods. The basic idea is very general and applies to any material with a yield criterion. It has already been suggested that the Spot Model could have relevance for glassy relaxation [27], and the SFR provides a general means to drive spot dynamics, based on solid mechanical principles. The Spot Model also provides a multiscale algorithm for random-packing dynamics, which works well for silo drainage [28], so the SFR could enable a general framework for multiscale modeling of amorphous materials. The idea would be to cycle between continuum stress calculations, meso-scale spot random walks, and microscopic particle dynamics.

\section{Acknowledgments}

The authors are gratefully indebted to the many researchers noted within who made their experimental data available for comparison with the present theory. Special thanks go to the members of GDR Midi (especially Olivier Pouliquen) and to J.-C. Tsai for their help in the transferring of data. The authors also thank Zdeněk P. Bažant for helpful comments on the manuscript, and Pierre Gremaud for providing Fig. 7

\section{APPENDIX A: CRITICAL STATE SOIL MECHANICS}

A common precept in plasticity is the notion of normality or associatedness. Flows that obey normality have a flow rule defined in terms of the yield function $Y$ as follows:

$$
\dot{\mathbf{E}}=\lambda \frac{\partial Y}{\partial \mathbf{T}}
$$

where $\lambda$ is a positive multiplier. For a $3 \mathrm{D}$ flow, this means that if the yield function were plotted in 6-space as a function of all 6 independent entries in the 3D stress tensor, the strain-rate matrix would be a 'vector' pointing normal to the yield surface oriented toward greater values of $Y$.

One of the first gripes about the use of friction-based yield criteria in describing granular materials is that the principle of normality gives a flow rule that predicts unstoppable dilatancy. Consider a rough extension of the Coulomb yield criterion into $3 \mathrm{D}, Y=\mu(\operatorname{tr} \mathbf{T}) / 3+$ $\left|\mathbf{T}_{\mathbf{0}}\right| / \sqrt{2}$, which displays the basic property that yield occurs when a certain multiple of the pressure equals the shear stress. Its associated flow rule is

$$
\dot{\mathbf{E}}=\lambda\left(\frac{\mathbf{T}_{\mathbf{0}}}{\sqrt{2}\left|\mathbf{T}_{\mathbf{0}}\right|}+\frac{\mu}{3} \mathbf{I}\right) .
$$


The trace of this strain-rate tensor is $\lambda \mu$, implying that material undergoing plastic flow will never stop dilating.

Roscoe and co-workers 89] present a different viewpoint on the issue. In what became known as Critical State Soil Mechanics, explained in detail in [39], they argue that normality does hold, but that in fact the Coulomb yield criterion is not technically the correct yield function.

Backed by results from triaxial stress experiments on soil samples, Critical State Theory claims that soils have a yield function that depends on the soil consolidation as measured by the local density $\rho$. The yield curve for material at a particular density is defined in terms of two stress tensor invariants: the pressure $p=-\frac{1}{3} \operatorname{tr} \mathbf{T}$ and the equivalent shear stress $q=\left|\mathbf{T}_{\mathbf{0}}\right| / \sqrt{2}$. Plotted in these variables, the principle of normality is equivalent to the statement that the strain-rate vector $(\dot{\epsilon}, \dot{\gamma})$ is normal to the yield curve and pointing outward, where $\dot{\epsilon}=-\operatorname{tr} \dot{\mathbf{E}}$ is a volumetric strain-rate which determines changes in density, and $\dot{\gamma}=\frac{\sqrt{2}}{3}\left|\dot{\mathbf{E}}_{\mathbf{0}}\right|$ is a shear strain-rate proportional to the total shear deformation (volume-conserving part of the deformation). Figure 26 displays the theory's picture of the yield function and how it changes after material deformation. Any stress state underneath the yield curve corrresponds to rigid material. Under normality, material at point 1 in the initial state will undergo a deformation according to the vector $\left(\dot{\epsilon}_{1}, \dot{\gamma}_{1}\right)$. Since $\dot{\epsilon}_{1}$ is negative, the material will dilate and settle down at point 1 on the right on a new yield curve corresponding to $\rho_{A}<\rho$. The material at stress state 2 will likewise undergo compaction and arrive at point 2 on the yield curve corresponding to $\rho_{B}>\rho$.

The critical state line is defined as the locus of points for which normality predicts no volumetric changes during deformation - note that wherever a yield curve intersects the critical state line, the curve becomes parallel to the $p$ axis and thus the corresponding strain-rate vector has no volumetric component. The theory reasons that the critical state line is indeed a straight line of the form

$$
q=M p
$$

As flow developes, the stress states throughout the material will continually move toward the critical state line and once local volume changes finally stop, all flowing material stress states should lie on the critical state line. Thus in a steady flow, the critical state line might falsely appear to be the yield function when in fact it is only a locus of states from a family of yield functions. So, it is argued then that the reason normality previously failed to describe granular materials was because it was applied mistakenly to the critical state line and not to the true family of yield functions.
[1] R. M. Nedderman, Statics and Kinematics of Granular Materials (Nova Science, 1991).

[2] V. V. Sokolovskii, Statics of Granular Materials (Pergamon/Oxford, 1965).

[3] R. Hill, The Mathematical Theory of Plasticity (Oxford at the Clarendon Press, 1950).

[4] H. M. Jaeger, S. R. Nagel, and R. P. Behringer, Rev. Mod. Phys. 68, 1259 (1996).

[5] P. G. de Gennes, Rev. Mod. Phys. 71, S374 (1999).

[6] L. P. Kadanoff, Rev. Mod. Phys. 71, 435 (1999).

[7] S. F. Edwards and D. V. Grinev, Advances in Complex Systems 4, 451 (2001), (also reprinted in Ref. 8]).

[8] T. Halsey and A. Mehta, eds., Challenges in Granular Physics (World Scientific, 2002).

[9] I. S. Aranson and L. S. Tsimring, Rev. Mod. Phys. 78, 641 (2006).

[10] C. S. Campbell, Ann. Rev. Fluid Mech. 22, 57 (1990).

[11] N. Balmforth and A. Provenzale, Geomorph. Fluid Mech. (Springer, 2001).

[12] J.-P. Bouchaud, M. E. Cates, J. R. Prakash, and S. F. Edwards, J. Phys. I (France) 4, 1383 (1994).

[13] J.-P. Bouchaud, M. E. Cates, J. R. Prakash, and S. F. Edwards, Phys. Rev. Lett. 74, 1982 (1995).

[14] T. Boutreux, E. Raphaël, and P.-G. de Gennes, Phys. Rev. E 58, 4692 (1998).

[15] T. Boutreux, H. A. Makse, and P.-G. de Gennes, Euro. Phys. J. B 9, 105 (1999).

[16] R. A. Bagnold, Proc. Roy. Soc. London Ser. A 225 (1954).
[17] D. Ertaş and T. C. Halsey, Europhys. Lett. 60, 931 (2002).

[18] S. B. Savage, J. Fluid Mech. 377, 1 (1998).

[19] W. Losert, L. Bocquet, T. C. Lubensky, and J. P. Gollub, Phys. Rev. Lett. 85, 1428 (2000).

[20] L. Bocquet, W. Losert, D. Schalk, T. C. Lubensky, and J. P. Gollub, Phys. Rev. E 65, 011307 (2002).

[21] P. Mills, D. Loggia, and M. Tixier, Europhys. Lett. 45, 733 (1999).

[22] O. Pouliquen and R. Gutfraind, Phys. Rev. E 53, 552 (1996).

[23] O. Pouliquen, Y. Forterre, and S. L. Dizes, Advances in Complex Systems 4, 441 (2001), (also reprinted in Ref. [8]).

[24] J. Litwiniszyn, Rheol. Acta 2/3, 146 (1958).

[25] J. Mullins, J. Appl. Phys. 43, 665 (1972).

[26] R. M. Nedderman and U. Tüzün, Powder Technology 22, 243 (1979).

[27] M. Z. Bazant, Mechanics of Materials 38, 717 (2006).

[28] C. H. Rycroft, M. Z. Bazant, G. S. Grest, and J. W. Landry, Physical Review E 73, 051306 (2006).

[29] A. Lemaitre, Phys. Rev. Lett. 89, 195503 (2002).

[30] A. Lemaitre, Phys. Rev. Lett. 89, 064303 (2002).

[31] I. S. Aranson and L. S. Tsimring, Phys. Rev. E 64, 020301 (2001).

[32] I. S. Aranson and L. S. Tsimring, Phys. Rev. E 65, 061303 (2002).

[33] G. D. R. Midi, Euro. Phys. Journ. E. 14, 341 (2004).

[34] M. Ostoja-Starzewski, Int. J. Plasticity 21, 1119 (2005). 

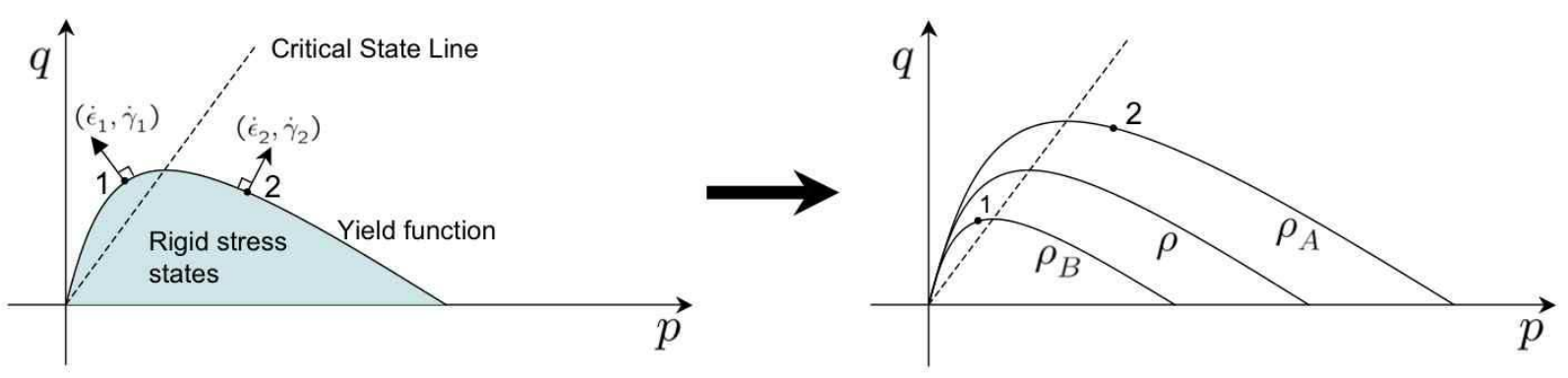

FIG. 26: (left) Critical State Theory's spade-shaped yield function for material at some density $\rho_{s}$; (right) Deformations with non-zero volumetric strain will cause the material to settle down on a new yield function.

[35] W. Prager and D. C. Drucker, Q. Appl. Mathematics 10:2, 157 (1952).

[36] R. M. Horne and R. M. Nedderman, Powder Technol. 14, $93(1976)$.

[37] E. B. Pitman, Powder Technol. 47, 219 (1986).

[38] P. A. Gremaud and J. V. Matthews, J. Comput. Phys. 166, 63 (2001).

[39] A. Schoefield and P. Wroth, Critical State Soil Mechanics (McGraw-Hill, 1968).

[40] A. Jenike, Journ. Appl. Mech. 31, 499 (1964).

[41] A. J. M. Spencer, J. Mech. Physics 12, 337351 (1964).

[42] L. Anand and C. Gu, J. Mech. Phys. Solids 28, 1701 (2000).

[43] A. Drescher, Analytical Methods in Bin-Load Analysis (Elsevier, 1991).

[44] E. B. Pitman and D. G. Schaeffer, Commun. Pure. Appl. Math. 40, 421 (1987).

[45] D. G. Schaeffer, J. Diff. Eq. 66, 19 (1987).

[46] A. Drescher, Phil. Trans. R. Soc. Lond. A 356, 2649 (1998).

[47] A. W. Jenike, Utah Engineering Experiment Station, Bulletin 108, University of Utah, Salt Lake City (1961).

[48] A. Samadani, A. Pradhan, and A. Kudrolli, Phys. Rev. E 60, 7203 (1999).

[49] J. Choi, A. Kudrolli, and M. Z. Bazant, J. Phys.: Condensed Matter 17, S2533 (2005).

[50] C. H. Rycroft, G. S. Grest, M. Z. Bazant, and J. W. Landry, Physical Review E p. to appear (2006).

[51] M. Jirásek and Z. P. Bažant, Inelastic Analysis of Structures (John Wiley and Sons Ltd., 2002).

[52] R. Hill, J. Mech. Phys. Solids 15, 79 (1967).

[53] U. Tüzün and R. M. Nedderman, Powder Technolgy 23, 257 (1979).

[54] A. Medina, J. A. Cordova, E. Luna, and C. Trevino, Physics Letters A 220, 111 (1998).

[55] D. Fenistein and M. van Hecke, Nature 425, 256 (2003).

[56] J. Litwiniszyn, Bull. Acad. Pol. Sci. 11, 593 (1963).

[57] J. Choi, A. Kudrolli, R. R. Rosales, and M. Z. Bazant, Phys. Rev. Lett. 92, 174301 (2004).

[58] H. Risken, The Fokker-Planck Equation (Springer, 1996).

[59] G. W. Baxter, R. P. Behringer, T. Fagert, and G. A. Johnson, Phys. Rev. Lett. 62, 2825 (1989).

[60] T. Pöschel, J. Phys. (France) I 4, 499 (1994).

[61] D. M. Mueth, Phys. Rev. E. 67, 011304 (2003).

[62] G. Lois, A. Lemaitre, and J. M. Carlson, Phys. Rev. E. 72, 051303 (2005).

[63] M. Latzel, S. Luding, H. J. Herrmann, D. W. Howell, and R. Behringer, Euro. Phys. Journ. E. 11, 325 (2003).
[64] F. da Cruz, Ph.D. thesis, Ecole Nationale des Ponts et Chaussees, Marne a la vallée, France (2004).

[65] L. Bocquet, W. Losert, D. Schalk, T. C. Lubensky, and J. P. Gollub, Phys. Rev. E 65, 011307 (2001).

[66] D. E. Mueth, G. F. Debregeas, G. S. Karczmar, P. J. Eng, S. R. Nagel, and H. M. Jaeger, Nature 406, 385 (2000).

[67] G. Chambon, J. Schmittbuhl, A. Corfdir, J. P. Vilotte, and S. Roux, Phys. Rev. E 68, 011304 (2003).

[68] S. Schöllmnann, Phys. Rev. E 59, 889 (1998).

[69] J.-C. Tsai and J. P. Gollub, Phys. Rev. E 70, 031303 (2004).

[70] J.-C. Tsai and J. P. Gollub, Phys. Rev. E 72, 051304 (2005).

[71] S. Siavoshi, A. V. Orpe, and A. Kudrolli, Phys. Rev. E 73, 010301(R) (2006).

[72] P. A. Thompson and G. S. Grest, Phys Rev Lett 67, 1751 (1991).

[73] D. Volfson, L. S. Tsimring, and I. S. Aranson, Physical Review E 68, 021301 (2003).

[74] P. Jalali, W. Polashenski, T. Tynjälä, and P. Zamankhan, Physica D 162, 188 (2002).

[75] cond-mat/0503682.

[76] P. A. Lemieux and D. J. Durian, Phys. Rev. Lett. 85, 4273 (2000).

[77] L. E. Silbert, J. W. Landry, and G. S. Grest, Phys. Fluids 15, 1 (2003).

[78] T. S. Komatsu, S. Inagaki, N. Nakagawa, and S. Nasuno, Phys. Rev. Lett. 86, 1757 (2001).

[79] L. E. Silbert, D. Ertas, G. S. Grest, T. C. Halsey, D. Levine, and S. J. Plimpton, Phys. Rev. E. 64, 051302 (2001).

[80] C. Ancey, Phys. Rev. E 65, 011304 (2002).

[81] G. Berton, R. Delannay, P. Richard, N. Taberlet, and A. Valance, Phys. Rev. E 68, 051303 (2003).

[82] O. Pouliquen, Phys. Fluids 11, 542 (1999).

[83] M. Prochnow, Ph.D. thesis, Ecole Nationale de Ponts et Chaussées, Marne la Vallée, France (2002).

[84] E. Azanza, Ph.D. thesis, Ecole des Ponts et Chaussées, Marne la Vallée, France (1997).

[85] F. da Cruz, S. Emam, M. Prochnow, J. Roux, and F. Chevoir, Phys. Rev. E. 72, 021309 (2005).

[86] J. Duran, Sands, Powders, and Grains (Springer-Verlag, 2000).

[87] G. I. Tardos, M. I. Khan, and D. G. Schaeffer, Phys. Fluids 10, 335 (1998).

[88] O. Pouliquen, Phys. Rev. Lett. 93, 248001 (2004).

[89] K. H. Roscoe, A. N. Schoefield, and A. Thurairajah, 
Geotechnique 15, 127 (1965). 